\title{
Distributed Optimization in an Energy-constrained Network
}

\author{
A DISSERTATION \\ SUBMITTED TO THE FACULTY OF THE GRADUATE SCHOOL \\ OF THE UNIVERSITY OF MINNESOTA \\ BY
}

Seid Alireza Razavi Majomard

IN PARTIAL FULFILLMENT OF THE REQUIREMENTS

FOR THE DEGREE OF

DOCTOR OF PHILOSOPHY

Professor Tom Luo, Adviser

February 2010 
(C) Seid Alireza Razavi Majomard February 2010 


\section{Acknowledgments}

My deepest gratitude and appreciation go to my Ph.D advisor, Professor Tom Luo, for his continuous academic support as well as financial assistance over my years in Ph.D program. I am greatly honored to work with Tom. His passion for science, great insights, and professional interactions made him my role model in the professional life.

I express my most sincere appreciation to my Master advisor, Professor Demoz Gebre-Egziabher, who also served in my Ph.D dissertation committee. I was fortunate to complete my master's degree under his supervision. He was an encouraging, inspiring and motivating force for me during my first years at University of Minnesota. Demoz, without your positive attitude, I would never be able reach this point in my life.

I am also deeply grateful to Professor Mostafa Kaveh, the head of my dissertation committee for his time and support through my years at University of Minnesota. Professor Kaveh has always been there; anytime I needed his help. Many thanks go to Professor Nihar Jindal who kindly accepted to serve on my examining committee and provided me helpful remarks and feedbacks.

My thanks go to my officemates Nick Kisialiou, Yao H Morin, and Meisam Razaviyayn for their help and the times we had at the office on the fourth floor. A special thanks goes to Professor Wenbo Zhang. I have enjoyed our collaboration and the time we have spent together. I am also thankful to Dr. Ramy Gohary and Dr. Tsung-Hui Chang.

My stay in Minnesota has been a unique experience in many aspects of my life. What made this experience magnificent was the support that I received from my wonderful friends in Minnesota: Shahrouz Takyar, Kiarash Bazargan, Haleh Hagh-Shenas, Siavash Pasalar, Hossein Zare, Rashad Moarref, Iman Ajang, Baktash Boghrati, Evan Ribnick, Parnell Mahoney, Tina Sarabandi and Ira Azakh. Without you, my graduate school years would have been tough!

Finally, I am eternally beholden to my wife Sarah who was and is my best friend, supporter, and 
critic. I am also deeply graceful to my parents Zahra and Mohammad, my brothers Mehdi and Ahmad. Words cannot express how much they helped me pursue my studies with their endless encouragement, unconditional love, and untold sacrifices. 
To my best friend: My wife Sarah Sharafkandi 


\begin{abstract}
We consider a distributed optimization problem whereby a network of $N$ nodes, $S_{\ell}$, $\ell \in\{1, \ldots, N\}$, wish to minimize a common strongly convex function $f(\mathbf{x}), \mathbf{x}=$ $\left[x_{1}, \ldots, x_{N}\right]^{T}$, under the constraint that node $S_{\ell}$ controls variable $x_{\ell}$ only. The nodes locally update their respective variables and periodically exchange their values over a set of pre-defined communication channels. Previous studies of this problem have focused mainly on the convergence issue and the analysis of convergence rate. In this work, we consider noisy communication channels and study the impact of communication energy on convergence. In particular, we study the minimum amount of communication energy required for nodes to obtain an $\epsilon$-minimizer of $f(\mathbf{x})$ in the mean square sense. For linear analog communication schemes, we prove that the communication energy to obtain an $\epsilon$-minimizer of $f(\mathbf{x})$ must grow at least at the rate of $\Omega(1 / \epsilon)$, and this bound is tight when $f$ is convex quadratic. Furthermore, we show that the same energy requirement can be reduced to $\mathcal{O}\left(\log ^{2} 1 / \epsilon\right)$ if suitably designed digital communication schemes are used.
\end{abstract}




\section{Contents}

List of Tables $\quad$ vii

List of Figures $\quad$ viii

Chapter 1 Introduction and Problem Statement 1

1.1 Related work . . . . . . . . . . . . . . . . 3

1.2 Problem statement $\ldots \ldots \ldots \ldots \ldots$

1.3 Summary of main contributions . . . . . . . . . . . . . 9

1.4 Organization of dissertation . . . . . . . . . . . . . . . 9

$\begin{array}{lll}\text { Chapter } 2 & \text { Analog Framework } & 11\end{array}$

2.1 Introduction . . . . . . . . . . . . . . . 11

2.1.1 Linear analog communication scheme . . . . . . . . . . 12

2.1.2 Local computation scheme . . . . . . . . . . . . . 14 
2.2 A necessary and sufficient convergence condition . . . . . . . . 15

2.3 Analytical lower bound on communication energy . . . . . . . . . . 18

2.4 Achieving the minimum energy bound . . . . . . . . . . . . . 19

2.5 Numerical validation . . . . . . . . . . . . . . 22

$\begin{array}{lll}\text { Chapter } 3 \text { Digital Framework } & 26\end{array}$

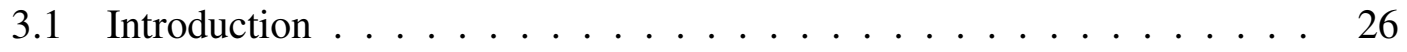

3.1.1 Digital communication scheme ............ 27

3.1.2 Local computation scheme . . . . . . . . . . . . 28

3.2 Convergence condition and communication energy . . . . . . . . 29

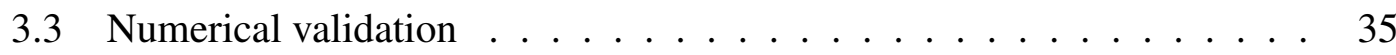

Chapter 4 Energy-distortion Trade-off in Digital and Analog Frameworks 37

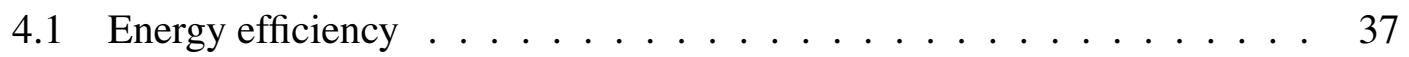

4.2 Simulation results ...................... 42

Chapter 5 Conclusion and Future Work 43

5.1 Conclusion .......................... 43

5.2 Directions for future work . . . . . . . . . . . . . . 44

$\begin{array}{ll}\text { Bibliography } & 47\end{array}$ 
$\begin{array}{lll}\text { Appendix B } & \text { Proof of Theorem 1 } & 61\end{array}$

Appendix C Proof of Lemma B.1 66

Appendix D $\quad$ Proof of (2.16) $\quad 71$

Appendix E Upper Bound on Communication Energy for the Isotropic Universal Design

$\begin{array}{lll}\text { Appendix F } & \text { Proof of Lemma C.1 } & 78\end{array}$

$\begin{array}{lll}\text { Appendix G } & \text { Proof of Lemma G.1 } & 80\end{array}$

$\begin{array}{lll}\text { Appendix H } & \text { Proof of Lemma } 1 & 82\end{array}$ 


\section{List of Tables}

2.1 Linear analog communication scheme . . . . . . . . . . . . . 12 


\section{List of Figures}

2.1 Mean squared error versus iteration number $(\beta=0.5) \ldots \ldots 22$

2.2 The average of the mean squared error $\times$ the communication energy

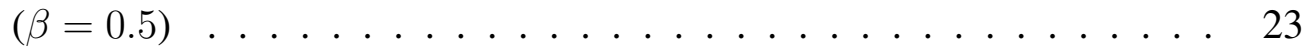

2.3 Mean squared error versus iteration number $(\kappa=4) \ldots . . . . . .24$

2.4 The average of the mean squared error $\times$ the communication energy

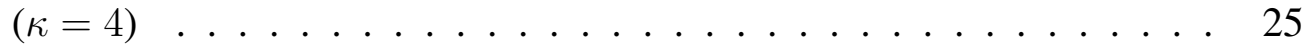

3.1 Mean squared error . . . . . . . . . . . . . 35

3.2 Normalized communication energy . . . . . . . . . . . . . 36

4.1 Mean squared error versus iteration number . . . . . . . . . . . 41

4.2 Mean squared error versus communication energy _. . . . . . . . 41 


\section{Chapter 1}

\section{Introduction and Problem Statement}

Distributed optimization where a network of nodes wish to minimize a common cost function has been studied extensively due to its applications in diverse areas including communication network, control theory, parallel computation and recently in wireless sensor network (WSN). A WSN is a wireless network consisting of spatially distributed sensor nodes, each with finite battery power and limited computational and mobility capability, which can cooperatively monitor and control physical or environmental conditions. Due to WSN's ability to withstand harsh environmental conditions, robustness to node failure, and unattended operations, the WSN becomes an attractive technology for situation awareness applications such as environmental monitoring and military surveillance. Examples of these applications include monitoring the state of permafrost in the Swiss Alps [1], the dynamics of glaciers in Briksdalsbreen in Norway [2], and detecting the enemy intrusion instead of using land-mines [3]. In addition, several standards are developed, or are under development for WSNs including the IEEE 1451 for the smart sensor market [4]. 
Wireless sensor networks are used broadly in two different configurations:

- Decentralized configuration (WSN with a fusion center): The fusion center is a special node (or set of nodes) with relatively high communication and computation capability and is responsible for generating the final estimation/detection results. In this setup, sensors typically communicate only with the fusion center and there is no inter-sensor communication.

- Distributed configuration: In this case, there is no fusion center and communication is peer to peer.

A major challenge in WSN with a fusion center is that transmitting data from each sensor node to a fusion center may require a significant amount of communication energy. Furthermore, decentralized configuration is not robust in case of fusion center failure. For these reasons, the most recent studies in WSNs consider a distributed configuration [5-11]. Distributed configuration in a WSN differs from the traditional central configuration in several important aspects:

- Sensor data is distributed across the network.

- Sensor nodes have limited computational capability and sensor operations are local and possibly asynchronous.

- Sensor nodes have limited energy and bandwidth for communication.

- Wireless channels can be fading or interfering with one another.

- The size and topology of a WSN may change unpredictably. 
Many problems in WSN can be formulated as a distributed optimization problems. For example, in the sensor localization problem, we are given the locations of anchor nodes and distance measurements between certain neighboring nodes in the network. The goal is to estimate the locations of all sensors in the network by distributed minimization of a cost function defined by a suitable norm of distance errors [12]. A special feature of this problem is the fact that nodes are usually battery operated and hence energy-constrained. Note that energy of each node is consumed for various operations including local computation and inter-sensor communication, with the latter being the dominant part. For example, the amount of energy required to transmit one bit for 200 meters range is enough for more than 800 thousands operations in Shockfish TinyNode 584 which is used for monitoring the state of permafrost in the Swiss Alps [13]. This motivates us to study the minimum amount of communication energy required for distributed optimization. In particular, we try to answer the question: "how much communication energy is needed to achieve a given level of solution accuracy in distributed optimization?"

\subsection{Related work}

The impact of channel noise and energy consumption on performance in WSNs with a fusion center is well-studied for decentralized estimation where energy-constrained sensor nodes wish to minimize a common distortion function. Examples of early work on decentralized estimation include the study in the context of distributed control $[14,15]$ and tracking [16], later in data fusion $[17,18]$, and most recently in wireless sensor networks $[19,20]$. These studies are focused on convergence behavior with some also making the additional assumption that the communication links are distortionless. The recent studies of decentralized estimation in sensor networks have considered the im- 
pact of channel noise and energy consumption on performance of estimation. From information-theoretic perspectives, the authors of [21-26] investigate the mean squared estimation error performance versus transmit power for the quadratic problem for the case of coherent multiple access channel. It is shown in [21] and [22] that if a WSN with AWGN channels is used to estimate a Gaussian source, a simple uncoded scheme outperforms the separate source-channel coding approach and leads to an optimal scaling behavior. Furthermore, it is proved that the uncoded communication scheme preserve the optimal scaling law in [25] for sensor networks with sensors ‘ output satisfying a certain mean condition, while the source-channel matching result is extended to more general homogeneous signal fields in [26]. If the sensor measurements are in a finite alphabet, type-based transmission schemes are proposed in [27-29]. The many-to-one transport capacity and compressibility are investigated for dense joint estimation sensor networks in [30], and an information-theoretic achievable rate-distortion region is derived in [31] when the communication links are unreliable.

For coherent multiple-access channels, it was shown in [32] that the linear estimation problem under bandwidth and power constraints can be formulated as a semi-definite programming (SDP) problem. The closed-form solutions for this SDP problem following the water-filling strategy is given in [33]. Among most of the existing studies, it is usually assumed that the joint distribution of the sensor observations is known. The universal signal processing algorithms that do not require the knowledge of the observation noise distributions are proposed in [34] and [35] for homogeneous and inhomogeneous sensor networks, respectively.

For the case of orthogonal channel usage between sensors and the fusion center, such as those in the existing multi-access communication schemes (TDMA/FDMA/CDMA), the 
linear decentralized estimation problem has been studied in [36-40]. It has been shown in [37] that the complexity of designing the optimal linear decentralized estimation is NP-hard. The suboptimal designs are provided in [38] using Gauss-Seidel algorithms by exploring the classic tools of canonical component analysis, and it is shown in [39, 40] that power scheduling, which is assigning most of the power to sensors with good observation and channel qualities and turning off sensors with lower quality, conserves energy.

A major challenge in WSN with a fusion center is that transmitting data from each sensor node to a fusion center may require a significant amount of communication energy. Furthermore, decentralized configuration is not robust in case of fusion center failure. For these reasons, the most recent studies consider a distributed setup in WSNs.

The convergence of distributed algorithms could be an issue especially when the communication channels are noisy. In this context, estimation of deterministic and random parameters in linear data models is considered in [5-11] using the notion of consensus averaging. The consensus averaging is also used for estimation of time-varying signals [41-44]. These studies are focused on convergence issues such as convergence rate and convergence conditions while some considering the effect of channel noise. Authors in [5] show that the consensus averaging schemes are eventually diverging if the communication channels are noisy [5]. As an alternative way to consensus averaging, $[45,46]$ uses the concept of mutually coupled oscillators and proposes a noise-robust algorithm with bounded, but not vanishing, mean squared error. Another noise-robust algorithm is developed in [11] using a single-hop communications between each sensor node and a bridge sensor node belonging to a special dominating set of communication graph. In addition, authors in [47] reformulate the sample mean estimator in a WSN as 
a distributed optimization problem and use an cyclic incremental gradient algorithm to derive noise-robust algorithm.

The effect of quantization noise on the convergence of distributed algorithms, such as when the digital communication are used, is studied in [48-51]. A bound on the divergence between quantized and unquantized consensus trajectories is provided in [48], and an algorithm is developed in [49] to mitigate the effect of quantization noise with predicting the neighbors' unquantized values. By careful accounting for round-off errors, authors in [50] propose an algorithm which converges to a quantized consensus. However, the quantized consensus is not a strict consensus. Self-imposed randomization in the quantization procedure, dithering, is used in [51] to propose an algorithm which converges to a strict consensus which might not be equal to the true value of network average.

In above studies of distributed optimization, the main topics are convergence issues such as the analysis of convergence and the rate of convergence and the impact of communication energy on convergence is not addressed. In this thesis, we study distributed optimization for sensor networks whereby the nodes are energy-constrained. In this context, we are naturally motivated to answer the question "how much energy is needed to achieve a given level of solution accuracy?". This question is perhaps more relevant than the question "how fast can an algorithm converge?", the latter being the main focus in the classical studies of distributed optimization $[52,53]$. In general, the communication energy is related to the amount of information exchange required among sensors as well as how accurate the inter-sensor communication must be. The minimum amount of information exchange required for distributed optimization was previously studied in [54]. However, unlike our current work, the communication channels were 
assumed distortion-less in [54], and there was no effort to characterize minimum energy consumption.

\subsection{Problem statement}

Let $\mathcal{F}_{M, L}$ be the set of continuously differentiable strongly convex functions $f(\mathbf{x})$ in the sense

$$
L\|\mathbf{x}-\mathbf{y}\|^{2} \leq\langle\nabla f(\mathbf{x})-\nabla f(\mathbf{y}), \mathbf{x}-\mathbf{y}\rangle \leq M L\|\mathbf{x}-\mathbf{y}\|^{2},
$$

for all $\mathbf{x}$ and $\mathbf{y}$ in the domain of $f(\mathbf{x})$, where $M$ and $L$ are positive numbers. Suppose a network of $n$ nodes, $S_{\ell}, \ell \in\{1, \ldots, n\}$ wish to jointly minimize a $f(\mathbf{x}) \in \mathcal{F}_{M, L}, \mathbf{x}=$ $\left[x_{1}, \ldots, x_{n}\right]^{T}$. Node $S_{\ell}$ only has control of variable $x_{\ell}$ and has the ability to compute the partial derivative $\partial f\left(\mathbf{x}^{\ell}\right) / \partial x_{\ell}$, where $\mathbf{x}^{\ell}$ is an estimate of the global variable $\mathbf{x}$ at node $S_{\ell}$

A special subclass of $\mathcal{F}_{M, L}$ is convex quadratic functions

$$
f(\mathbf{x})=\frac{1}{2} \mathbf{x}^{T} \mathbf{A} \mathbf{x}+\mathbf{b}^{T} \mathbf{x}+c
$$

where $\mathbf{A}$ is a symmetric positive definite matrix, $\mathbf{b} \in \mathbb{R}^{n}$, and $c$ is a constant value. In this case, $L=\lambda_{\min }(\mathbf{A})$ (the minimum eigenvalue) and $M=\operatorname{cond}(\mathbf{A})$ (the condition number). Moreover, if node $S_{\ell}$ knows the $\ell$-th row of matrix $\mathbf{A}$ and the $\ell$-th element of vector $\mathbf{b}$, then $S_{\ell}$ can compute the partial derivative of $f(\mathbf{x})$ with respect to its local variable $x_{\ell}$.

Neighboring nodes in the network communicate through orthogonal time-invariant noisy channels. The communication channel between nodes $S_{\ell}$ and $S_{j}$ is corrupted by additive 
noise, $n_{\ell, j}(t)$, with zero mean and variance $\sigma_{\ell, j}^{2}$. In this model, the received signal by node $S_{j}$ from $S_{\ell}$, is given as

$$
r_{j, \ell}(t)=y_{\ell}(t)+n_{\ell, j}(t)
$$

where $y_{\ell}(t)$ is the signal transmitted by node $S_{\ell}$ at iteration $t$ to its neighbors (multicast). In this way, communication energy at iteration $t$ for node $S_{\ell}$ is proportional to $\mathbb{E}\left[\left|y_{\ell}(t)\right|^{2}\right]$.

This thesis aims to study the minimum communication energy required to obtain an $\epsilon$ minimizer of $f(\mathbf{x})$ in the mean square sense. A random vector $\mathbf{x}$ is an $\epsilon$-minimizer of $f(\mathbf{x})$ in the mean square sense if

$$
\mathbb{E}\left[\left\|\mathbf{x}-\mathbf{x}^{*}\right\|^{2}\right] \leq \epsilon, \mathbf{x}^{*}=\arg \min _{x} f(\mathbf{x})
$$

A distributed optimization problem of this kind arises naturally in sensor network applications. For example, in the sensor localization problem, we are given the locations of anchor nodes and distance measurements between certain neighboring nodes in the network. The goal is to estimate the locations of all sensors in the network by distributed minimization of a cost function $f(\mathbf{x})$ defined by a suitable norm of distance errors [12]. In this context, $x_{\ell}$ is the location of sensor $S_{\ell}$ and is to be estimated by $S_{\ell}$. Since sensor nodes are usually battery operated, it is essential that the algorithms for local computation and inter-sensor communication are designed to be energy efficient. This motivates us to study distributed optimization strategies which require minimum amount of communication energy, the latter being a dominant part of each sensor's energy consumption. 


\subsection{Summary of main contributions}

For a class of linear analog messaging schemes, we study distributed stochastic gradient type algorithms $[55,56]$, and derive a necessary and sufficient condition for convergence in the mean square sense. We prove that the communication energy required to obtain an $\epsilon$-minimizer of $f(\mathbf{x})$ must grow at least at the rate of $\Omega(1 / \epsilon)$. In addition, we introduce a specific design which attains this minimum energy bound within a constant factor when $f(\mathbf{x})$ is convex quadratic. The proposed algorithms for local computation and communication are isotropic and universal in the sense that they are independent of node index and problem data. These features can simplify the deployment and management of networks.

In addition, we consider a class of digital communication schemes and assess their energy efficiency in the context of distributed optimization. Using a suitably designed digital communication scheme, we show that the distributed gradient projection method [54] requires only $\mathcal{O}\left(\log ^{2} 1 / \epsilon\right)$ communication energy to find an $\epsilon$-minimizer of a strongly convex function. This is a significant reduction from $\mathcal{O}(1 / \epsilon)$ communication energy required for analog schemes. Moreover, since gradient projection method converges linearly as opposed the sub-linear convergence of the stochastic gradient type algorithms, the former will require fewer iterations to find an $\epsilon$-minimizer (at least asymptotically), and is therefore likely to consume less energy in local computation as well.

\subsection{Organization of dissertation}

The dissertation is organized as follows. In Chapter 2, we study distributed optimization using linear analog communication schemes. We will focus on the class of convex 
quadratic function, a sub class of strongly convex functions. We first prove that the communication energy required to obtain an $\epsilon$-minimizer of convex quadratic function must grow at least at the rate of $\Omega(1 / \epsilon)$. We then introduce a isotropic universal design that attains this lower bound within a constant factor. The study of the distributed optimization using digital communication schemes are presented in Chapter 3. In this chapter, we introduce a distributed algorithm which obtains an $\epsilon$-minimizer of strongly convex function by spending $\mathcal{O}\left(\log ^{2} 1 / \epsilon\right)$ communication energy. In chapter 4 , we compare energy-distortion trade-off in digital and analog frameworks. In particular, we we explain why the digital framework is more energy-efficient than the analog framework for distributed optimization. Finally, Chapter 5 gives the conclusions and some directions for future work. 


\section{Chapter 2}

\section{Analog Framework}

\subsection{Introduction}

In this chapter, we consider distributed optimization using analog communication schemes and prove that the communication energy grows at least at the rate of $\Omega(1 / \epsilon)$ to obtain an $\epsilon$-minimizer of convex functions. In fact, we show that $\Omega(1 / \epsilon)$ lower-bound holds for strongly convex quadratic functions. Moreover, We provide a specific design which attains this minimum energy bound within a factor of at most 3 for convex quadratic functions. The proposed design is isotropic and universal in the sense that the local computation in each node is independent of node index and problem data. These features greatly simplify the deployment and management of networks.

Through this chapter, we focus on class of convex quadratic functions which is a subclass of strongly convex functions. Consider a general convex quadratic cost function

$$
f(\mathbf{x})=\frac{1}{2} \mathbf{x}^{T} \mathbf{A} \mathbf{x}+\mathbf{b}^{T} \mathbf{x}+c
$$




\begin{tabular}{c|ll}
$y_{\ell}(t)$ & Convergence & Communication energy \\
\hline$x_{\ell}^{\ell}(t)$ & yes & high \\
$x_{\ell}^{\ell}(t)-x_{\ell}^{\ell}(t-1)$ & no & - \\
\hline \hline $\mathbf{x}_{\ell}^{\ell}(\mathbf{t})-\gamma(\mathbf{t}) \mathbf{x}_{\ell}^{\ell}(\mathbf{t}-\mathbf{1})$ & possible & $\lim _{t \rightarrow \infty} \gamma(t) \rightarrow 1$
\end{tabular}

Table 2.1: Linear analog communication scheme

where $\mathbf{x}=\left[x_{1}, \ldots, x_{n}\right]^{T}, \mathbf{A} \in \mathbb{R}^{n \times n}, \mathbf{A} \succ \mathbf{0}$, and $\mathbf{b} \in \mathbb{R}^{n}, c \in \mathbb{R}$. Notice that

$$
\lambda_{\min } \leq \frac{\langle\nabla f(\mathbf{x})-\nabla f(\hat{\mathbf{x}}), \mathbf{x}-\hat{\mathbf{x}}\rangle}{\|\mathbf{x}-\hat{\mathbf{x}}\|^{2}} \leq \operatorname{cond}(\mathbf{A}) \lambda_{\min }
$$

where $\lambda_{\min }$ and cond $(\mathbf{A})$ are minimum eigenvalue and the condition number of matrix A, respectively. Therefore, convex quadratic cost function $f(\mathbf{x})$ belongs to the set $\mathcal{F}_{\text {cond }(\mathbf{A}), \lambda_{\min }}$. Assume node $S_{\ell}$ knows the $\ell$-th row of matrix $\mathbf{A}$ and $\ell$-th element of vector $\mathbf{b}$, denoted by $[\mathbf{A}]_{\ell}$ and $b_{\ell}$, respectively. In this setup, $[\mathbf{A}]_{\ell}$ and $b_{\ell}$ represent local information between $S_{\ell}$ and its neighbors. Thus, node $S_{\ell}$ is able to compute the partial derivative of $f(\mathbf{x})$ with respect to its local variable $x_{\ell}$.

We introduce a distributed optimization algorithm whereby each node iteratively updates its local variable and tracks the variable values of its neighbors using a linear analog communication scheme.

\subsubsection{Linear analog communication scheme}

After each local update, node $S_{\ell}$ should relay its information to its neighbors. One way is to directly send the updated value of its local variable, $x_{\ell}^{\ell}(t)$. This results in a communication energy consumption of $\mathbb{E}\left[\left|x_{\ell}^{\ell}(t)\right|^{2}\right]$ at iteration $t$ which tends to the constant 
value $\left|x_{\ell}^{*}\right|^{2}$ if the iterates converge to $\mathbf{x}^{*}$ in the mean square sense. An alternative way is to send the incremental value, $x_{\ell}^{\ell}(t)-x_{\ell}^{\ell}(t-1)$, in which case the communication energy, $\mathbb{E}\left[\left|x_{\ell}^{\ell}(t)-x_{\ell}^{\ell}(t-1)\right|^{2}\right]$, would vanish as $x_{\ell}^{\ell}$ converges to $x_{\ell}^{*}$. In general, we can consider a linear analog communication scheme (table 2.1) where the transmitted signal, $y_{\ell}(t)$, is a linear combination of $x_{\ell}^{\ell}(t), x_{\ell}^{\ell}(t-1)$, namely

$$
y_{\ell}(t)=\psi_{\ell}(t)\left[x_{\ell}^{\ell}(t)-\gamma_{\ell}(t) x_{\ell}^{\ell}(t-1)\right]
$$

In equation (2.2), the amplification factor $\psi_{\ell}(t)$ and coefficient $\gamma_{\ell}(t)$ are design parameters $\left(0 \leq \gamma_{\ell}(t) \leq 1\right)$ that are independent of $f(\mathbf{x})$. Receiver node, $S_{j}$, tracks its neighbors' variables according to

$$
\begin{aligned}
x_{\ell}^{j}(t) & =\gamma_{\ell}(t) x_{\ell}^{j}(t-1)+\frac{r_{j, \ell}(t)}{\psi_{\ell}(t)} \\
& =x_{\ell}^{\ell}(t)+\gamma_{\ell}(t)\left(x_{\ell}^{j}(t-1)-x_{\ell}^{\ell}(t-1)\right)+\frac{n_{\ell, j}(t)}{\psi_{\ell}(t)},
\end{aligned}
$$

where we have replaced the received message $r_{j, \ell}(t)$ by (1.3). It follows that, for all $t \geq 1$,

$$
x_{\ell}^{j}(t)-x_{\ell}^{\ell}(t)=\gamma_{\ell}(t)\left(x_{\ell}^{j}(t-1)-x_{\ell}^{\ell}(t-1)\right)+\frac{n_{\ell, j}(t)}{\psi_{\ell}(t)},
$$

which, by recursion, implies

$$
x_{\ell}^{j}(t)-x_{\ell}^{\ell}(t)=\sum_{i=1}^{t} \prod_{k=i+1}^{t} \gamma_{\ell}(k) \frac{n_{\ell, j}(i)}{\psi_{\ell}(i)}
$$

Notice that the total communication energy up to iteration $t$ is

$$
E_{\text {com }}(t)=\sum_{i=1}^{t} \sum_{\ell=1}^{n} \mathbb{E}\left[\left|\psi_{\ell}(i)\left[x_{\ell}^{\ell}(i)-\gamma_{\ell}(i) x_{\ell}^{\ell}(i-1)\right]\right|^{2}\right] .
$$




\subsubsection{Local computation scheme}

Optimization in the presence of noise can be carried out by the stochastic gradient type algorithms [55]. Each iteration of this algorithm is given as

$$
\mathbf{x}(t)=\mathbf{x}(t-1)-a(t) \mathbf{g}(\mathbf{x}(t-1)), t=1, \ldots
$$

where $a(t)$ is a sequence of vanishing positive step sizes satisfying

$$
\sum_{t=1}^{\infty} a(t)=\infty, \quad \sum_{t=1}^{\infty} a^{p}(t)<\infty, p \geq 2,
$$

and $\mathbf{g}(\mathbf{x}(t))$ is a noisy version of the gradient vector $\nabla f(\mathbf{x}(t))$. We consider a distributed implementation of (2.4) whereby $S_{j}$ uses the noisy copy of its neighbors' variables to compute an estimate of $\partial f(\mathbf{x}(t)) / \partial x_{j}$ (denoted by $\left.g_{j}(\mathbf{x}(t))\right)$, and update $x_{j}^{j}$ by

$$
x_{j}^{j}(t)=x_{j}^{j}(t-1)-\frac{1}{t^{\beta}} g_{j}\left(x_{1}^{j}(t-1), \ldots, x_{n}^{j}(t-1)\right),
$$

where we have chosen $a(t)=1 / t^{\beta}$, with $0<\beta<1$.

For the convex quadratic function (1.2), the distributed algorithm described by (2.3)(2.5) can be summarized in vector form as

$$
\mathbf{x}(t)=\mathbf{x}(t-1)-\frac{1}{t^{\beta}}(\mathbf{A x}(t-1)+\mathbf{b}+\mathbf{v}(t-1)),
$$


where $\mathbf{x}(t) \triangleq\left[x_{1}^{1}(t), \ldots, x_{n}^{n}(t)\right]^{T}$, and

$$
\mathbf{v}(t) \triangleq\left[\begin{array}{c}
\sum_{\ell=1}^{n} a_{1, \ell} \sum_{i=1}^{t} \prod_{k=i+1}^{t} \gamma_{\ell}(k) \frac{n_{\ell, 1}(i)}{\psi_{\ell}(i)} \\
\vdots \\
\sum_{\ell=1}^{n} a_{n, \ell} \sum_{i=1}^{t} \prod_{k=i+1}^{t} \gamma_{\ell}(k) \frac{n_{\ell, n}(i)}{\psi_{\ell}(i)}
\end{array}\right]
$$

where $a_{m, \ell}$ denotes the $(m, \ell)$-th entry of $\mathbf{A}$. For notational convenience, we define $n_{\ell, \ell}(i) \triangleq 0$.

Clearly, both the communication energy and the algorithm convergence depend on the choice of parameters $\left\{\gamma_{\ell}(t), \psi_{\ell}(t): t=1,2, \ldots\right\}$. Our goal is to determine these parameters which will minimize the total communication energy while still ensuring the algorithm convergence.

\subsection{A necessary and sufficient convergence condition}

We consider convex quadratic function (1.2) in this section. We first derive a necessary and sufficient convergence condition of the distributed algorithm described by (2.3)(2.5) and then derive a lower bound on communication energy. Define

$$
\alpha_{\ell}(i, t, q) \triangleq \sum_{j=i+1}^{t} \frac{1}{j^{\beta}} \prod_{k=j+1}^{t}\left(1-\frac{\lambda_{q}}{k^{\beta}}\right) \prod_{k=i+1}^{j-1} \gamma_{\ell}(k)
$$

where $\lambda_{q}$ is an eigenvalue of matrix $\mathbf{A}$.

Proposition 1 The iterates $\{\mathbf{x}(t)\}$ generated by the distributed algorithm $(2.3)-(2.5)$ converge to the minimizer of (1.2) in the mean square sense for all positive definite 
matrix $\mathbf{A}$ if and only if

$$
\lim _{t \rightarrow \infty} \sum_{i=1}^{t-1}\left(\frac{\alpha_{\ell}(i, t, q)}{\psi_{\ell}(i)}\right)^{2}=0, \quad \forall \ell \in\{1, \ldots, n\}, \forall \lambda_{q}>0 .
$$

Proof: We first use (2.6) to compute the mean squared error at $t$-th iteration, and then show that it converges to zero under condition (2.8).

Define the error at the $t$-th iteration as $\mathbf{e}(t) \triangleq \mathbf{x}(t)-\mathbf{x}^{*}$. It follows from (2.6) that

$$
\begin{aligned}
\mathbf{e}(t) & =\left(\mathbf{I}-\frac{\mathbf{A}}{t^{\beta}}\right) \mathbf{e}(t-1)+\frac{\mathbf{v}(t-1)}{t^{\beta}} \\
& =\mathbf{e}_{i n}(t)+\mathbf{e}_{c h}(t),
\end{aligned}
$$

where, by recursion,

$$
\begin{aligned}
& \mathbf{e}_{i n}(t) \triangleq \prod_{i=1}^{t}\left(\mathbf{I}-\frac{\mathbf{A}}{i^{\beta}}\right) \mathbf{e}(0), \\
& \mathbf{e}_{c h}(t) \triangleq \sum_{i=1}^{t} \frac{1}{i^{\beta}} \prod_{j=i+1}^{t}\left(\mathbf{I}-\frac{\mathbf{A}}{j^{\beta}}\right) \mathbf{v}(i-1) .
\end{aligned}
$$

In equation (2.9), $\mathbf{e}_{i n}(t)$ and $\mathbf{e}_{c h}(t)$ represent the errors due to initialization and channel noise, respectively.

The initial error, $\mathbf{e}(0)$, and the accumulated channel noise, $\mathbf{v}(i), i \in\{1, \ldots, t-1\}$ are uncorrelated. Therefore, the mean squared error at the $t$-th iteration, from (2.9), is

$$
\mathbb{E}\left[\|\mathbf{e}(t)\|^{2}\right]=\left\|\mathbf{e}_{i n}(t)\right\|^{2}+\mathbb{E}\left[\left\|\mathbf{e}_{c h}(t)\right\|^{2}\right] .
$$


The distributed algorithm (2.3)-(2.5) converges to $\mathrm{x}^{*}$ in the mean square sense if and only if both terms in the right-hand side of (2.10) vanish as $t \rightarrow \infty$. It is shown in Appendix A that

$$
\begin{gathered}
\left\|\mathbf{e}_{i n}(t)\right\|^{2}=\mathcal{O}\left(\exp \left(\frac{-2 \lambda_{\min }(t+1)^{1-\beta}}{1-\beta}\right)\right), \\
\mathbb{E}\left[\left\|\mathbf{e}_{c h}(t)\right\|^{2}\right]=\sum_{m, q, \ell=1}^{n} p_{m, q}^{2} a_{m, \ell}^{2} \sigma_{\ell, m}^{2} \sum_{i=1}^{t-1}\left(\frac{\alpha_{\ell}(i, t, q)}{\psi_{\ell}(i)}\right)^{2},
\end{gathered}
$$

where $\lambda_{\min }$ is the minimum eigenvalue of the matrix $\mathbf{A}, p_{m, q}$ is the $(m, q)$-th element of the matrix $\mathbf{P}$ (the orthonormal matrix of eigenvalue decomposition of the matrix $\mathbf{A}$ ), $a_{m, \ell}$ is the $(m, \ell)$-th element of the matrix $\mathbf{A}$, and $\sigma_{\ell, m}^{2}$ is the variance of channel noise $n_{\ell, m}(j)$.

Since matrix $\mathbf{A}$ is a positive definite matrix, the minimum eigenvalue of the matrix $\mathbf{A}$ is positive, and thus,

$$
\lim _{t \rightarrow \infty}\left\|\mathbf{e}_{i n}(t)\right\|^{2}=0
$$

Therefore, the distributed algorithm (2.3)-(2.5) converges to $\mathrm{x}^{*}$ in the mean square sense if and only if

$$
\lim _{t \rightarrow \infty} \mathbb{E}\left[\left\|\mathbf{e}_{c h}(t)\right\|^{2}\right]=0
$$

for all $\mathbf{P}$ and $\sigma_{m \ell}^{2}$, or equivalently,

$$
\lim _{t \rightarrow \infty} \sum_{i=1}^{t-1}\left(\frac{\alpha_{\ell}(i, t, q)}{\psi_{\ell}(i)}\right)^{2}=0, \quad \forall \ell \in\{1, \ldots, n\}, \forall \lambda_{q}>0
$$


Proposition 1 is proven.

Proposition 1 implies that the distributed algorithm (2.3)-(2.5) obtains an $\epsilon$-minimizer of $f(\mathbf{x})$, as long as parameters $\left\{\gamma_{\ell}(t), \psi_{\ell}(t): t=1,2, \ldots\right\}$ are properly chosen.

\subsection{Analytical lower bound on communication energy}

Theorem 1 Consider the distributed algorithm described by (2.3) - (2.5). Then, there holds

$$
\liminf _{t \rightarrow \infty} \mathbb{E}\left[\|\mathbf{e}(t)\|^{2}\right] E_{\text {com }}(t)>0
$$

The detailed proof is given in Appendix B. Below, we provide a proof sketch. Similar to (2.10) for the mean squared error, we can show that the communication energy is sum of two terms: the communication energy for the noise-free scenario $\left(E_{i n}(t)\right)$ and another term due to the channel noise. Recall from (2.10) that $\mathbb{E}\left[\|\mathbf{e}(t)\|^{2}\right] \geq \mathbb{E}\left[\left\|\mathbf{e}_{c h}(t)\right\|^{2}\right]$ and, thus, we have

$$
\begin{aligned}
\mathbb{E}\left[\|\mathbf{e}(t)\|^{2}\right] E_{\text {com }}(t) & \geq \mathbb{E}\left[\left\|\mathbf{e}_{c h}(t)\right\|^{2}\right] E_{i n}(t) \\
& =\sum_{\ell=1}^{n} \mathbb{E}\left[e_{c h, \ell}^{2}(t)\right] \sum_{\ell=1}^{n} E_{i n, \ell}(t) \\
& \geq \sum_{\ell=1}^{n} \mathbb{E}\left[e_{c h, \ell}^{2}(t)\right] E_{i n, \ell}(t),
\end{aligned}
$$

where $\mathbb{E}\left[e_{c h, \ell}^{2}(t)\right]$ is the contribution of channel noise between node $S_{\ell}$ and its neighbors in the mean squared error, and $E_{i n, \ell}(t)$ is the amount of energy spent by node $S_{\ell}$ in 
noise-free scenario. Using the Cauchy-Schwartz inequality and the fact that algorithm is convergent in the mean squared sense, we derive the asymptotic lower-bound on each summand. We have

$$
\liminf _{t \rightarrow \infty} \mathbb{E}\left[\|\mathbf{e}(t)\|^{2}\right] E_{c o m}(t) \geq \sum_{m, q, \ell=1}^{n} \frac{a_{m, \ell}^{2} p_{m, q}^{2} \sigma_{\ell, m}^{2}\left|x_{\ell}^{*}\right|^{2}}{2 \lambda_{q}^{2}}
$$

The right-hand side of (2.13) is a positive number for non-zero optimal point $\mathrm{x}^{*}$.

For an isotropic design where each node operates identically, $\left(\psi_{\ell}(i)=\psi(i), \gamma_{\ell}(i)=\right.$ $\gamma(i))$, we can prove that

$$
\liminf _{t \rightarrow \infty} \mathbb{E}\left[\|\mathbf{e}(t)\|^{2}\right] E_{c o m}(t) \geq \sum_{m, q, \ell=1}^{n} \frac{a_{m, \ell}^{2} p_{m, q}^{2} \sigma_{\ell, m}^{2}\left\|\mathbf{x}^{*}\right\|^{2}}{2 \lambda_{q}^{2}}
$$

following the same steps of the proof of Theorem 1.

Theorem 1 shows that the communication energy required to obtain an $\epsilon$-minimizer of $f(\mathbf{x})$ must grow at least at the rate of $\Omega(1 / \epsilon)$.

\subsection{Achieving the minimum energy bound}

We will prove that the isotropic universal design with

$$
\begin{aligned}
& \hat{\gamma}(i) \triangleq\left(1-i^{-1}\right)^{\kappa}, \\
& \hat{\psi}(i) \triangleq i^{\frac{1+\kappa}{2}}
\end{aligned}
$$

and $\kappa>2(1-\beta)>0$, attains the minimum energy bound within a constant factor. We first show that the parameters $\left\{\hat{\psi}_{\ell}(i), \hat{\gamma}_{\ell}(i), i=1, \ldots, t\right\}$ satisfy the convergence condition (2.8). We then prove that communication energy is inversely proportional to 
the mean squared error.

In a isotropic design, the necessary and sufficient condition for convergence is given as

$$
\lim _{t \rightarrow \infty} \sum_{i=1}^{t-1} \frac{\alpha^{2}(i, t, q)}{i^{1+\kappa}}=0, \quad \forall \ell \in\{1, \ldots, n\}, \forall \lambda_{q}>0
$$

For the choice (2.15), an upper bound on $\alpha(i, t, q)$ is derived in Appendix D:

$$
\alpha(i, t, q) \leq\left(\frac{2}{\lambda_{q}(t-1)^{\kappa}}+e^{\frac{-\lambda_{q}(t+1)^{1-\beta}}{1-\beta}}\right) i^{\kappa}
$$

Therefore, we have

$$
\begin{aligned}
\sum_{i=1}^{t-1} \frac{\alpha^{2}(i, t, q)}{i^{1+\kappa}} & \leq\left(\frac{2}{\lambda_{q}(t-1)^{\kappa}}+e^{\frac{-\lambda_{q}(t+1)^{1-\beta}}{1-\beta}}\right)^{2} \sum_{i=1}^{t-1} i^{\kappa-1} \\
& \leq \frac{4}{\lambda_{q}^{2} \kappa(t-1)^{\kappa}}+o\left(t^{-\kappa}\right)
\end{aligned}
$$

Equation (2.17) shows that the design in (2.15) satisfies the convergence condition (2.8). Since the contribution of initial error in the mean squared error $\left\|\mathbf{e}_{i n}(t)\right\|^{2}$ vanishes exponentially (2.11), the mean squared error is bounded by

$$
\mathbb{E}\left[\|\mathbf{e}(t)\|^{2}\right] \leq \sum_{m, q, \ell=1}^{n} \frac{4 a_{m, \ell}^{2} p_{m, q}^{2} \sigma_{\ell, m}^{2}}{\lambda_{q}^{2} \kappa(t-1)^{\kappa}}+o\left(t^{-\kappa}\right),
$$

by substituting (2.17) in (2.12). Equation (2.18) shows that the proposed algorithm has a sub-linear convergence rate.

An upper bound on communication energy is derived in Appendix E:

$$
E_{\text {com }}(t) \leq \frac{9}{2} \kappa\left\|\mathbf{x}^{*}\right\|^{2} t^{\kappa}+o\left(t^{\kappa}\right)
$$


We conclude from (2.18) and (2.19) that

$$
\lim _{t \rightarrow \infty} \mathbb{E}\left[\|\mathbf{e}(t)\|^{2}\right] E_{c o m}(t) \leq \sum_{m, q, \ell=1}^{n} \frac{18 a_{m, \ell}^{2} p_{m, q}^{2} \sigma_{\ell, m}^{2}\left\|\mathbf{x}^{*}\right\|^{2}}{\lambda_{q}^{2}}
$$

or equivalently,

$$
\mathbb{E}\left[\|\mathbf{e}(t)\|^{2}\right] E_{\text {com }}(t)=\mathcal{O}(1)
$$

It has been shown in section 2.2 that

$$
\mathbb{E}\left[\|\mathbf{e}(t)\|^{2}\right] E_{\text {com }}(t)=\Omega(1)
$$

Therefore, the communication energy is inversely proportional to the mean squared error asymptotically. Moreover, the design in (2.15) requires a communication energy that is within a constant factor of the minimum communication energy.

Corollary 1 The distributed algorithm described by (2.3) - (2.5) with parameters in (2.15) consumes energy not more than 36 times of the minimum communication energy in the class of isotropic universal designs.

Proof: Divide the upper bound on communication energy for the proposed design (2.20) by the lower bound on communication energy (2.14) to obtain

$$
\frac{\lim _{t \rightarrow \infty} E_{\text {com }}(t)}{\liminf _{t \rightarrow \infty} E_{\text {com }}(t)} \leq 36
$$

Corollary 1 is proven. 


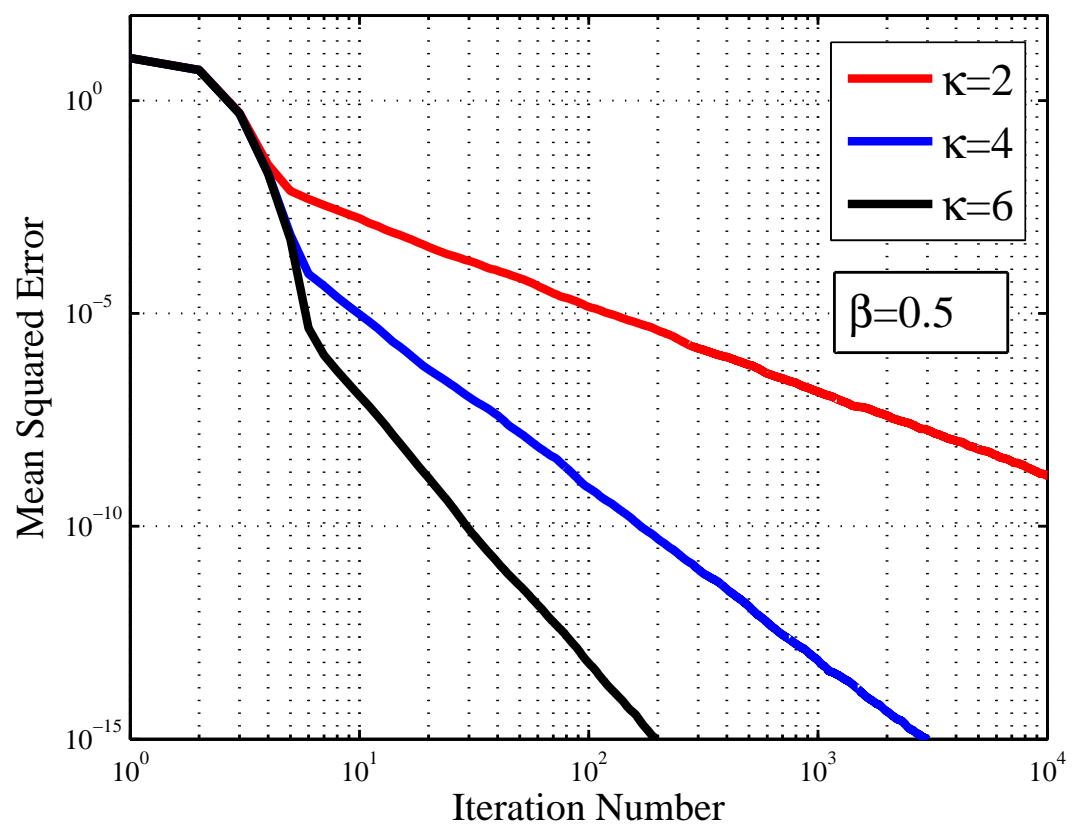

Figure 2.1: Mean squared error versus iteration number $(\beta=0.5)$

\subsection{Numerical validation}

We have shown in the pervious section that the margin between the minimum communication energy and the communication energy in the proposed isotropic universal design is independent of the choice of the power of step size $\beta$ and variable $\kappa$. In this section, we present numerical simulations to illiterate this fact and study the effects of $\beta$ and $\kappa$ on the convergence behavior of the proposed algorithm. We consider a scenario where 10 nodes collaborate to minimize a common quadratic convex function. Matrix A is randomly generated, and we assume the minimum point and initial point at $\mathbf{x}^{*}=[1, \ldots, 1]^{T}$ and $\mathbf{x}(1)=[0, \ldots, 0]^{T}$, respectively. The communication channels are additive white gaussian channels, and the channel noise variances $\sigma_{m, \ell}^{2}$ are taken to be $\sigma_{m, \ell}^{2}=1+Z_{m, \ell}$, where $Z_{m, \ell}=Z_{\ell, m}$ are i.i.d. random variables with $Z_{m, \ell} \sim \chi_{1}^{2}$. We use stochastic gradient type algorithm in (2.5) with different power of step size $\beta$ and the 


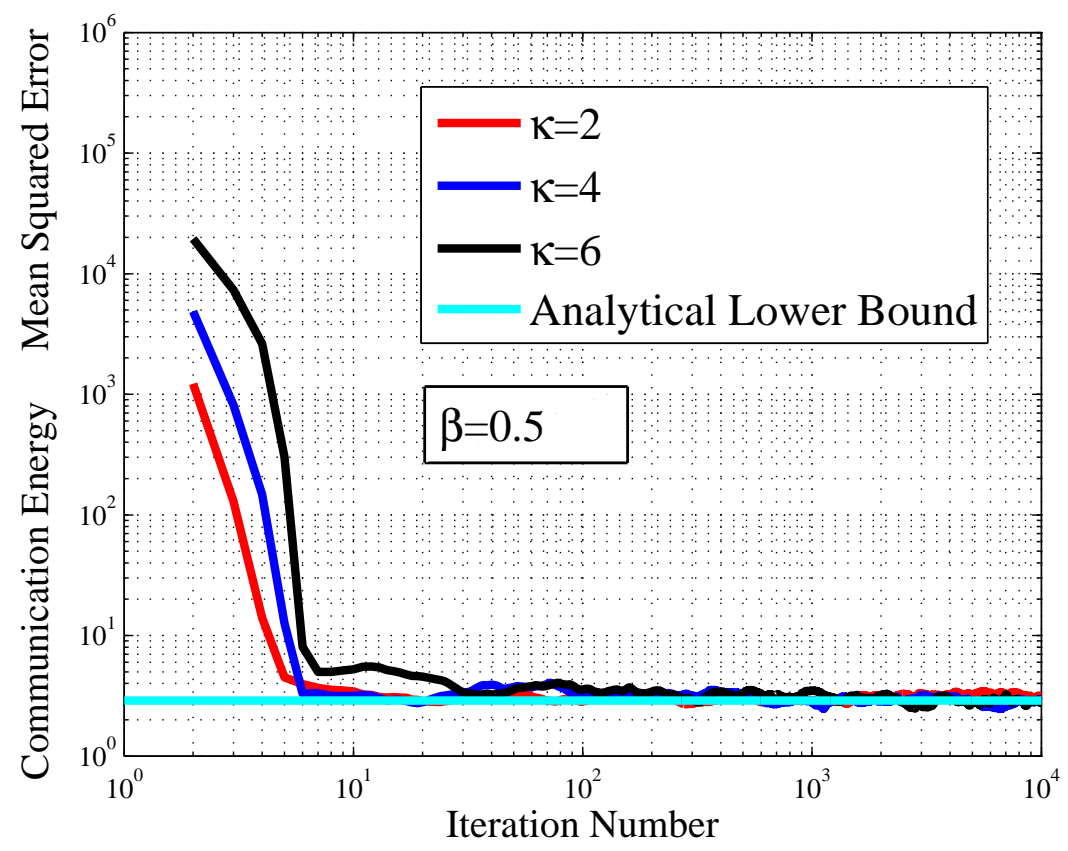

Figure 2.2: The average of the mean squared error $\times$ the communication energy $(\beta=$ $0.5)$

universal design in (2.15) with different values of $\kappa$. Each figure presents the simulation results averaged over 50 trails.

We first study the effect of $\kappa$ while the step size $\beta$ is fixed $(\beta=0.5)$. Figure 2.1 verifies that the mean squared error decreases at the rate of $t^{-\kappa}$ after the accumulated channel noise becomes the dominant source of error. However, a higher convergence rate also requires a higher communication energy such that the product of the communication energy and mean squared error is lower bounded by a constant value as it is shown in Figure 2.2. In other words, the communication energy required to compute $\epsilon$-minimizer of $f(\mathbf{x})$ in mean square sense grows at the rate of $\Theta(1 / \epsilon)$. These results agree with our theoretical analysis presented in pervious sections. Moreover, Figure 2.2 suggests that the analytical lower bound on the product of the communication energy and mean 


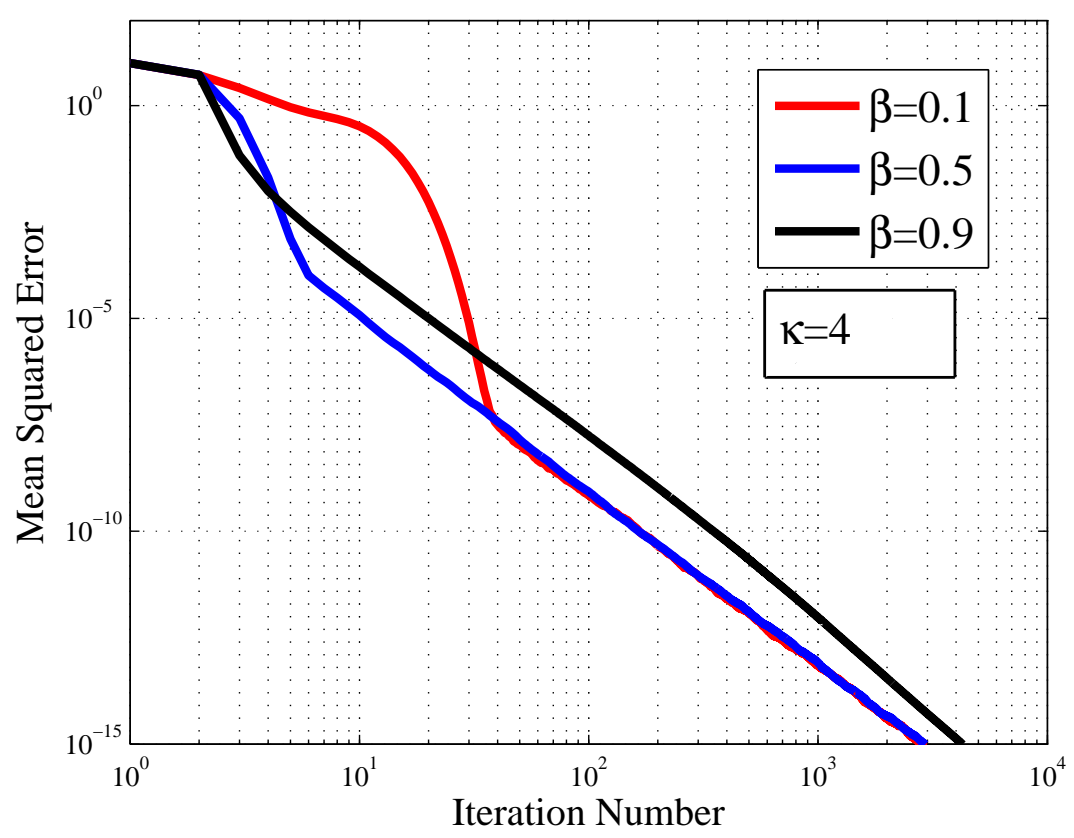

Figure 2.3: Mean squared error versus iteration number $(\kappa=4)$

squared error (2.14) is tight.

We then investigate the effect of step sizes with fixed $\kappa(\kappa=4)$. Figure 2.3 shows that the contribution of initial error in the mean squared error is decreasing slowly while the step size is sharply vanishing $(\beta=0.9)$, thus the product of the communication energy and mean squared error slowly converges to its lower bound (Figure 2.4). On the other hand, when the step size is slowly vanishing $(\beta=0.1)$, The mean squared error vanishes slowly in the initial steps of the algorithm, resulting a higher value of the product of the mean squared error and communication energy. 


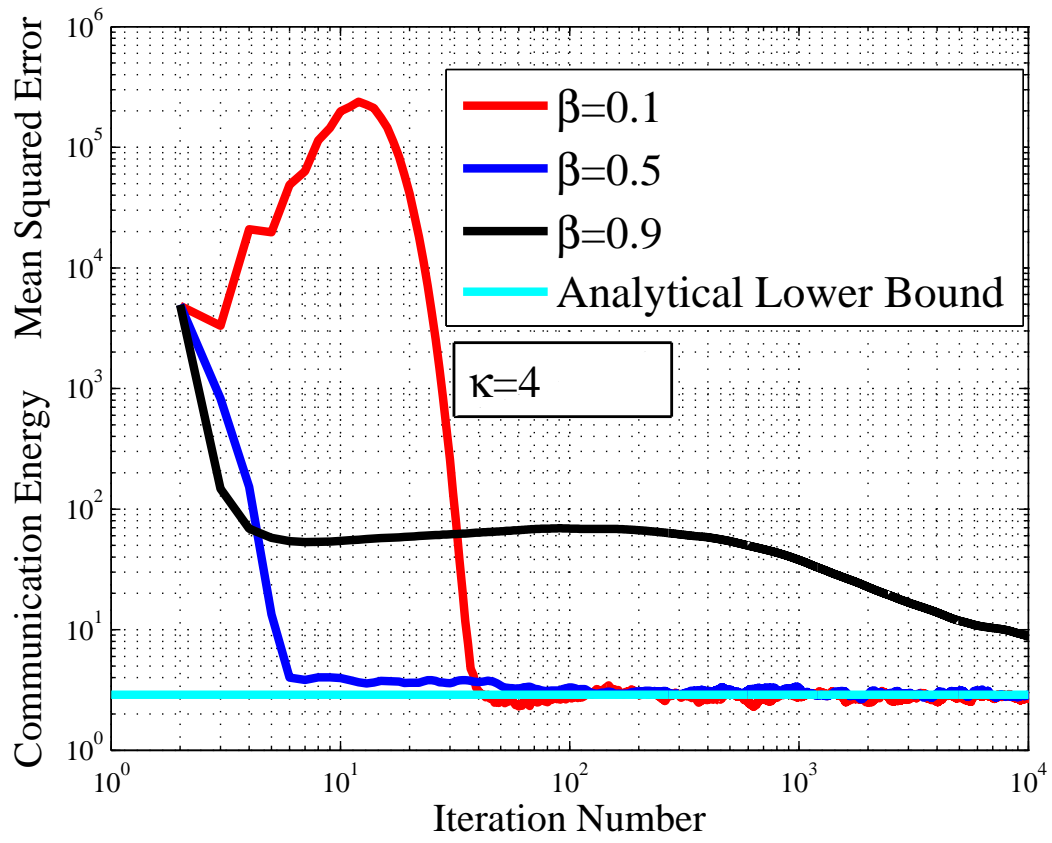

Figure 2.4: The average of the mean squared error $\times$ the communication energy $(\kappa=4)$ 


\section{Chapter 3}

\section{Digital Framework}

\subsection{Introduction}

In pervious chapter, we considered analog communication schemes for the distributed optimization problem and proved that the communication energy grows at the rate of $\Omega(1 / \epsilon)$ in order to obtain an $\epsilon$-minimizer of a strongly convex function. In this section, we introduce a distributed algorithm based on a digital communication scheme which requires $\mathcal{O}\left(\log ^{2} 1 / \epsilon\right)$ communication energy to obtain an $\epsilon$-minimizer of a strongly convex function. We introduce a distributed optimization algorithm whereby each node iteratively updates its local variable and tracks the variable values of its neighbors using a digital communication scheme. 


\subsubsection{Digital communication scheme}

Similar to the analog communication scheme, node $S_{\ell}$ uses a linear messaging scheme where the transmitted signal, $m_{\ell}(t)$, is given as

$$
m_{\ell}(t)=\mathcal{Q}\left(x_{\ell}^{\ell}(t)-\gamma(t) x_{\ell}^{\ell}(t-1)\right)=\left[\operatorname{sgn}_{\ell}^{\ell}(t), \operatorname{amp}_{\ell}^{\ell}(t)\right],
$$

where $\operatorname{sgn}_{\ell}^{\ell}(t)$ represents the sign of $x_{\ell}^{\ell}(t)-\gamma(t) x_{\ell}^{\ell}(t-1)$, and $\operatorname{amp}_{\ell}^{\ell}(t)$ is the binary representation of integer part of $2^{R(t)}\left|x_{\ell}^{\ell}(t)-\gamma(t) x_{\ell}^{\ell}(t-1)\right|$. The resolution of quantizer $R(t)$ is a design parameter and will be determined later. Receiver node $S_{j}$ first detects $m_{\ell}^{j}(t)=\left[\operatorname{sgn}_{\ell}^{j}(t), \operatorname{amp}_{\ell}^{j}(t)\right]$ and then reconstructs $x_{\ell}^{j}$ as following $\left(x_{\ell}^{j}(0)=0\right)$

$$
x_{\ell}^{j}(t)=\gamma(t) x_{\ell}^{j}(t-1)+\operatorname{sgn}_{\ell}^{j}(t) 2^{-R(t)} \operatorname{amp}_{\ell}^{j}(t) .
$$

Notice that variable $x_{\ell}^{j}(t)$ at node $S_{j}$ is a noisy copy of node $S_{\ell}$ 's variable $x_{\ell}^{\ell}(t)$. Define $n_{Q, \ell}(t)$, and $e_{\ell, j}(t)$ as quantization noise at node $S_{\ell}$ at iteration $t$, and communication error due to channel noise between node $S_{\ell}$ and $S_{j}$ at iteration $t$, respectively.

$$
\begin{gathered}
n_{Q, \ell}(t) \triangleq \operatorname{sgn}_{\ell}^{\ell}(t) 2^{-R(t)} \operatorname{amp}_{\ell}^{\ell}(t)-\left(x_{\ell}^{\ell}(t)-\gamma(t) x_{\ell}^{\ell}(t-)\right), \\
e_{\ell, j}(t) \triangleq \operatorname{sgn}_{\ell}^{j}(t) 2^{-R(t)} \operatorname{amp}_{\ell}^{j}(t)-\operatorname{sgn}_{\ell}^{\ell}(t) 2^{-R(t)} \operatorname{amp}_{\ell}^{\ell}(t) .
\end{gathered}
$$

It follows from (3.2) that

$$
\begin{aligned}
x_{\ell}^{j}(t) & =\gamma(t) x_{\ell}^{j}(t-1)+x_{\ell}^{\ell}(t)-\gamma(t) x_{\ell}^{\ell}(t-1)+n_{Q, \ell}(t)+e_{\ell, j}(t) \\
& =x_{\ell}^{\ell}(t)+\gamma(t)\left(x_{\ell}^{j}(t-1)-x_{\ell}^{\ell}(t-1)\right)+n_{Q, \ell}(t)+e_{\ell, j}(t)
\end{aligned}
$$




$$
=x_{\ell}^{\ell}(t)+\sum_{i=1}^{t} \prod_{k=i+1}^{t} \gamma(k)\left(n_{Q, \ell}(i)+e_{\ell, j}(i)\right) .
$$

\subsubsection{Local computation scheme}

Optimization in the presence of noise can be carried out by the gradient projection algorithm proposed in [54]. One iteration of this algorithm is given as

$$
\mathbf{x}(t)=[\mathbf{x}(t-1)-a \mathbf{g}(\mathbf{x}(t-1))]_{+}, \mathbf{x}(0)=0 .
$$

where $[x]_{+}$is the projection of $x$ on $[0,1], a$ is a constant positive step size, and $\mathbf{g}(\mathbf{x}(t))$ is a noisy version of the gradient vector $\nabla f(\mathbf{x}(t))$. For $f(\mathbf{x}) \in \mathcal{F}_{M, L}$, it is proven in [54] that if $\mathbf{g}(\mathbf{x}(t))$ satisfies

$$
\|\mathbf{g}(\mathbf{x}(t))-\nabla f(\mathbf{x}(t))\| \leq n^{1 / 2} \alpha^{t}, t=1, \ldots,
$$

for $\alpha$ sufficiently close to 1 and less than 1 , then the iterates $\{\mathbf{x}(t)\}$ generated by the gradient projection algorithm (3.4) converges linearly to the optimal point. We consider a distributed implementation of the gradient projection algorithm whereby $S_{j}$ uses a noisy copy of its neighbors' variables to compute an estimate of $\partial f(\mathbf{x}(t)) / \partial x_{j}$ (denoted by $\left.g_{j}(\mathbf{x}(t))\right)$, and update $x_{j}^{j}$ by $\left(x_{j}^{j}(0)=0\right)$

$$
x_{j}^{j}(t)=\left[x_{j}^{j}(t-1)-\frac{1}{L M} g_{j}\left(x_{1}^{j}(t), \ldots, x_{n}^{j}(t)\right)\right]_{+} .
$$

Here, we have chosen the step size to be $a=\frac{1}{M L}$. In the next section, we first derive a sufficient condition on the coefficient $\gamma(t)$, the resolution $R(t)$, and probability of bit error $P_{b}(t)$ to obtain an $\epsilon$-minimizer of $f(\mathbf{x})$. We then bound the total communication 
energy.

\subsection{Convergence condition and communication energy}

Let $\mathbf{x}(t)=\left[x_{1}^{1}(t), \ldots, x_{n}^{n}(t)\right]^{T}$ and define $\mathbf{g}(\mathbf{x}(t))$ as a vector of the partial derivatives of $f(\mathbf{x})$ with respect to the local variables which are computed locally at each node using the noisy replicas of neighbors variables:

$$
\mathbf{g}(\mathbf{x}(t)) \triangleq\left[g_{1}\left(x_{1}^{1}(t), \ldots, x_{n}^{1}(t)\right), \ldots, g_{n}\left(x_{1}^{n}(t), \ldots, x_{n}^{n}(t)\right)\right]^{T}
$$

Then, the distributed computation (3.6) can be expressed as the gradient projection algorithm

$$
\mathbf{x}(t)=\left[\mathbf{x}(t-1)-\frac{1}{L M} \mathbf{g}(\mathbf{x}(t-1))\right]_{+} .
$$

Notice that the convergence condition (3.5) is not necessarily satisfied for every realization of channel noise. Therefore, the sequence $\{\mathbf{x}(t)\}$ generated by (3.7) might not deterministically converge to the optimal point. However, under a modified assumption, the sequence $\{\mathbf{x}(t)\}$ converges to the optimal point in the mean square sense.

Lemma 1 For $f(\mathbf{x}) \in \mathcal{F}_{M, L}$, if $\mathbf{g}(\mathbf{x}(t))$ satisfies

$$
\mathbb{E}\left[\|\mathbf{g}(\mathbf{x}(t))-\nabla f(\mathbf{x}(t))\|^{2}\right] \leq n \alpha^{2 t}, t=1, \ldots,
$$

then the sequence $\{\mathrm{x}(t)\}$ generated by the gradient projection algorithm (3.7) converges linearly to the optimal point in the mean square sense such that

$$
\mathbb{E}\left[\left\|\mathbf{x}(t)-\mathbf{x}^{*}\right\|^{2}\right] \leq \frac{2 A}{L} n \alpha^{2 t}
$$


for constants $A$ and $\alpha$ satisfying

$$
\left(1-\frac{1}{M}\right)+\frac{1}{L M A}+\left(1+\frac{1}{M}\right) \sqrt{\frac{2}{A L}} \leq \alpha^{2}<1 .
$$

The proof of Lemma 1 is similar to the proof of proposition 5.1 in [54] and presented in Appendix H. We use Lemma 1 to show that the proposed algorithm generates an $\epsilon$-minimizer of $f(\mathbf{x})$ if the design parameters are chosen as

$$
\begin{aligned}
\gamma(t) & =\alpha, \\
R(t) & =\left[\log _{2}\left(\sqrt{\frac{2 M L}{1-\alpha^{2}}} \alpha^{-2 t}\right)\right], \\
P_{b}(t) & =\frac{3\left(1-\alpha^{2}\right)}{8 L M} \alpha^{4 t},
\end{aligned}
$$

where $\lceil x\rceil$ is the smallest integer number greater than $x, \alpha$ is a positive constant less than 1 .

Theorem 2 If the design parameters, $\gamma(t), R(t)$, and $P_{b}(t)$ satisfy (3.10)-(3.12), then the distributed algorithm described by (3.1), (3.2), and (3.6) converges to the global minimum of $f(\mathbf{x}) \in \mathcal{F}_{M, L}$ over $[0,1]^{n}$ in the mean square sense. Moreover, the communication energy to obtain an $\epsilon$-minimizer of $f(\mathbf{x}), \mathbf{x}\left(t_{\epsilon}\right)$, is

$$
E_{\text {com }}\left(t_{\epsilon}\right)=\mathcal{O}\left(\log ^{2} 1 / \epsilon\right)
$$


Proof: We first show that the convergence condition (3.8) of Lemma 1 holds. Therefore, the proposed algorithm obtains an $\epsilon$-minimizer of $f(\mathbf{x})$.

It follows form the definition of strongly convex functions (1.1) that

$$
\|\nabla f(\mathbf{x})-\nabla f(\hat{\mathbf{x}})\| \leq M L\|\mathbf{x}-\hat{\mathbf{x}}\|
$$

Therefore, we have

$$
\begin{aligned}
\mathbb{E}\left[\|\mathbf{g}(\mathbf{x}(t))-\nabla f(\mathbf{x}(t))\|^{2}\right] & \leq M L \mathbb{E}\left[\sum_{\ell=1}^{n}\left(x_{\ell}^{J}(t)-x_{\ell}^{\ell}(t)\right)^{2}\right] \\
& =M L \sum_{\ell=1}^{n} \mathbb{E}\left[\left(x_{\ell}^{J}(t)-x_{\ell}^{\ell}(t)\right)^{2}\right]
\end{aligned}
$$

where $J$ is given as

$$
J=\arg \max _{j}\left\|\left[x_{1}^{j}(t)-x_{1}^{1}(t), \ldots, x_{n}^{j}(t)-x_{n}^{n}(t)\right]^{T}\right\|^{2}
$$

Substitute the difference between variable $x_{\ell}^{\ell}$ and its noisy copy at node $S_{J}, x_{\ell}^{J}$, from equation (3.3), and use the fact that the quantization noise and communication error are uncorrelated to obtain

$$
\begin{aligned}
\mathbb{E}\left[\left(x_{\ell}^{J}(t)-x_{\ell}^{\ell}(t)\right)^{2}\right] & =\mathbb{E}\left[\left(\sum_{i=1}^{t} \prod_{k=i+1}^{t} \gamma(k)\left(n_{Q, \ell}(i)+e_{\ell, J}(i)\right)\right)^{2}\right] \\
& =\sum_{i=1}^{t} \prod_{k=i+1}^{t} \gamma^{2}(k)\left(\mathbb{E}\left[n_{Q, \ell}^{2}(i)\right]+\mathbb{E}\left[e_{\ell, J}^{2}(i)\right]\right) .
\end{aligned}
$$


The power of quantization noise $\mathbb{E}\left[n_{Q, \ell}^{2}(i)\right]$ is upper-bounded by

$$
\mathbb{E}\left[n_{Q, \ell}^{2}(i)\right] \leq 2^{-2 R(i)},
$$

where $R(i)$ is resolution of quantizer. Notice that the number of transmitted bits at $\mathrm{i}$-th iteration is bounded by $R(i)$. Therefore, the power of communication error $\mathbb{E}\left[e_{\ell, J}^{2}(i)\right]$ is upper-bounded by

$$
\mathbb{E}\left[e_{\ell, J}^{2}(i)\right] \leq \sum_{q=0}^{R(i)} P_{b}(i) 2^{-2 q} \leq \frac{4 P_{b}(i)}{3}
$$

where $P_{b}(i)$ is bit error probability of channel at iteration $i$. Substitute (3.14)-(3.16) into (3.13) to derive

$$
\mathbb{E}\left[\|\mathbf{g}(\mathbf{x}(t))-\nabla f(\mathbf{x}(t))\|^{2}\right] \leq n M L \sum_{i=1}^{t} \prod_{k=i+1}^{t} \gamma^{2}(k)\left(2^{-2 R(i)}+\frac{4 P_{b}(i)}{3}\right)
$$

It follows from Lemma 1 that the proposed algorithm converges to the global optimum of $f(\mathbf{x})$ over $[0,1]^{n}$ if

$$
\sum_{i=1}^{t} \frac{\left(2^{-2 R(i)}+\frac{4 P_{b}(i)}{3}\right)}{\prod_{k=1}^{i} \gamma^{2}(k)} \leq \frac{\alpha^{2 t}}{M L \prod_{k=1}^{t} \gamma^{2}(k)}, t=1, \ldots
$$

Notice the left-hand side of the inequality (3.17) is a non-decreasing function of iteration number $t$. We select $\gamma(k)$ such that the right-hand of the inequality (3.17) also be a nondecreasing function of iteration number. Therefore, we have $\alpha^{2} / \gamma^{2}(t) \geq 1$, and thus $\gamma(t) \leq \alpha$. Let $\gamma(t)=\alpha$. Then, to prove the convergence of the proposed algorithm, it is 
enough to show that

$$
\begin{aligned}
\sum_{i=1}^{t} \alpha^{-2 i} 2^{-2 R(i)} & \leq \frac{1}{2 M L} \\
\sum_{i=1}^{t} \alpha^{-2 i} P_{b}(i) & \leq \frac{3}{8 M L}
\end{aligned}
$$

Recall the resolution $R(i)$ from (3.11) and notice that $\alpha<1$. Therefore, the inequality (3.18) holds as

$$
\sum_{i=1}^{t} \alpha^{-2 i} 2^{-2 R(i)} \leq \frac{1-\alpha^{2}}{2 L M} \sum_{i=1}^{t} \alpha^{2 i} \leq \frac{1}{2 L M}
$$

Similarly, the inequality (3.19) holds for the choice of the bit error probability $P_{b}(i)$ at (3.12). Therefore, the convergence condition (3.8) is satisfied and the algorithm converges linearly to the optimal point in the mean square sense (Lemma 1). In particular, the algorithm obtains an $\epsilon$-minimizer of $f(\mathbf{x})$ at iteration $t_{\epsilon}$, if

$$
t_{\epsilon} \geq\left\lceil\frac{1}{2} \log _{\alpha}\left(\frac{L \epsilon}{2 A n}\right)\right\rceil
$$

So far, we have shown that the proposed algorithm obtains an $\epsilon$-minimizer of $f(\mathbf{x})$. Next, we derive the communication energy in terms of $\epsilon$.

Using M-ary orthogonal communication schemes such as multiple frequency-shift keying (MFSK), the energy per bit $W_{\ell}\left(P_{b}\right)$ is upper bounded by [57]:

$$
W_{\ell}\left(P_{b}\right) \leq \frac{2 \max _{j} N_{\ell, j}}{s_{\ell}} \log \left(\frac{1}{2 P_{b}\left(1-2^{-s_{\ell}}\right)}\right)+2 \max _{j} N_{\ell, j} \log (2)
$$


where $s_{\ell}$ is the number of bits per symbol which is transmitted by node $S_{\ell}, \frac{1}{2} N_{\ell, j}$ is the channel noise variance between nodes $S_{\ell}$ and $S_{j}$, and $P_{b}$ is the required bit error probability. Therefore, the total communication energy is

$$
E_{\text {com }}(t)=\sum_{i=1}^{t} \sum_{\ell=1}^{n} W_{\ell}\left(P_{b}(i)\right) b_{\ell}(i)
$$

where $b_{\ell}(i)$ is the number of bits in the message $m_{\ell}(i)$. The communication energy per symbol is upper bounded by

$$
W_{\ell}\left(P_{b}(i)\right) b_{\ell}(i) \leq 2 \max _{j} N_{\ell, j}\left(\log \left(\frac{1}{2 P_{b}(i)\left(1-2^{-b_{\ell}(i)}\right)}\right)+\log (2) b_{\ell}(i)\right)
$$

Here, the number of bits per symbol is equal to the number of transmitted bit at each iteration $\left(s_{\ell}=b_{\ell}(i)\right)$. The number of transmitted bits at $i$-th iteration $b_{\ell}(i)$ is bounded by $R(i)+1$, where the resolution $R(i)$ grows linearly with iteration number. Substitute the resolution $R(i)$ and probability of bit error $P_{b}(i)$ from equations (3.11-3.12) into (3.21) to obtain

$$
W_{\ell}\left(P_{b}(i)\right) b_{\ell}(i) \leq 12 \max _{j} N_{\ell, j} \log (1 / \alpha) i+\mathcal{O}(1)
$$

Therefore, the total communication energy is

$$
E_{\text {com }}\left(t_{\epsilon}\right) \leq \sum_{i=1}^{t_{\epsilon}}\left(c_{1} i+\mathcal{O}(1)\right)=\frac{c_{1}}{2} t_{\epsilon}^{2}+\mathcal{O}\left(t_{\epsilon}\right)
$$

where constant $c_{1}$ is equal to $12 n \max _{j, \ell} N_{\ell, j} \log (1 / \alpha)$. Replace the iteration number from (3.20) into (3.22) to derive 


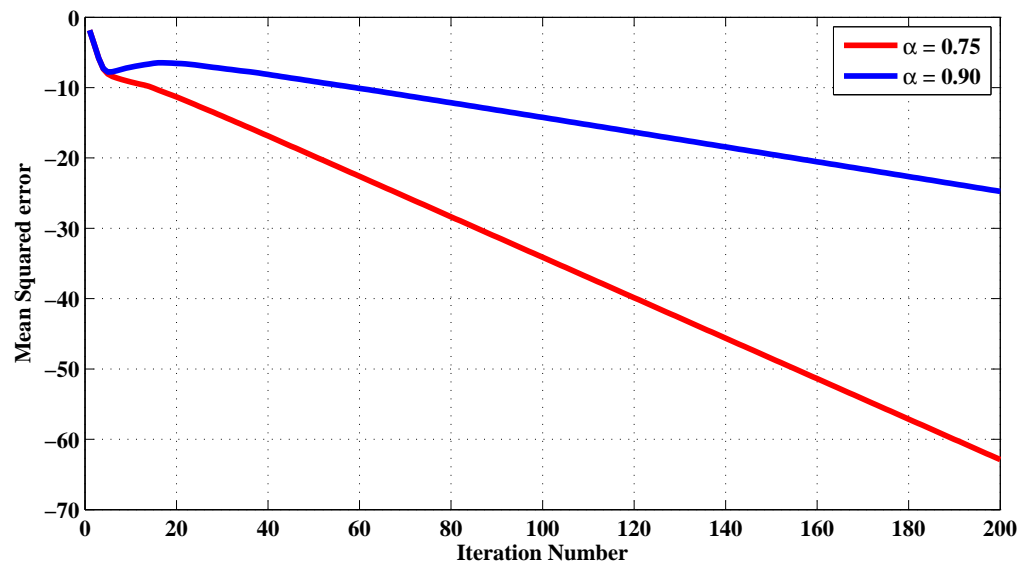

Figure 3.1: Mean squared error

$$
E_{\text {com }}\left(t_{\epsilon}\right)=c_{2} \log ^{2} 1 / \epsilon+\mathcal{O}(\log 1 / \epsilon)
$$

where constant $c_{2}$ is equal to $\frac{c_{1}}{8 \log ^{2} 1 / \alpha}$. The theorem is proven.

\subsection{Numerical validation}

To illustrate the theoretical results, we consider a simple example where 10 nodes collaborate to minimize a quadratic convex function

$$
f(\mathbf{x})=\frac{1}{2} \mathbf{x}^{T} \mathbf{A} \mathbf{x}+\mathbf{b}^{T} \mathbf{x}+\mathbf{c},
$$

where $\mathbf{A} \in \Re e^{10 \times 10}, \mathbf{A} \succ 0$. Notice that $f(\mathbf{x}) \in \mathcal{F}_{M, L}$ where $M$ and $L$ are the condition number and minimum eigenvalue of matrix $\mathbf{A}$, respectively. We generate a random matrix A such that $f(\mathbf{x}) \in \mathcal{F}_{2,1}$, and select vector $\mathbf{b}$ such that $f(\mathbf{x})$ has a minimum point at $\mathbf{x}=[0.5, \ldots, 0.5]^{T}$. Since all coefficients $w_{\ell}$ are scaled by a common factor. In sim- 


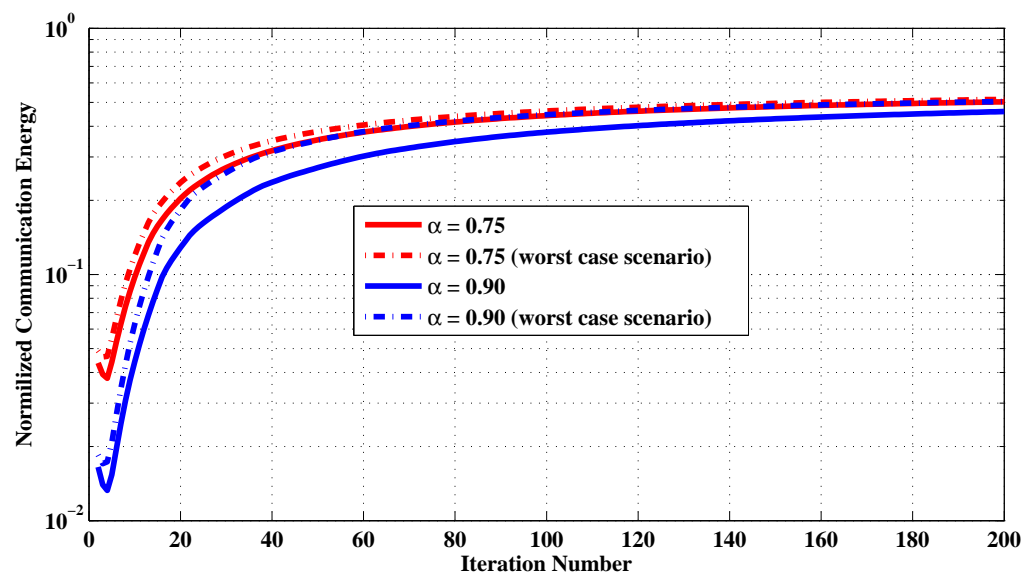

Figure 3.2: Normalized communication energy

ulation, $w_{\ell}$ are taken to be maximum of channel path loss $w_{\ell}=\max _{j} d_{\ell, j}^{\kappa}, \kappa=2$. The mean square error for different value of $\alpha$ averaged over 10 runs is shown in Figure 3.1. This figure confirms that the algorithm converges linearly to the optimal point. Notice that the choice of $\alpha$ should satisfy (3.9). In particular, $\alpha \geq \sqrt{1-1 / M}=\sqrt{2} / 2$. Figure 3.2 shows that the communication energy required to obtain $\epsilon$-minimizer, normalized by $c_{2} \log ^{2} 1 / \epsilon$, is bounded. This figure also shows the normalized communication energy for the worst case scenario where $R(i)+1$ number of bits is transmitted by each node at i-th iteration. These results agree with our theoretical analysis. 


\section{Chapter 4}

\section{Energy-distortion Trade-off in Digital and Analog Frameworks}

In the pervious chapters, we study the impact of communication energy on convergence in distributed optimization using both the linear analog and digital communication scheme. The goal of this chapter is to compare the trade-off between communication energy and the mean squared error in digital and analog frameworks. Furthermore, we explain why the digital framework is more energy-efficient than the analog framework for distributed optimization.

\subsection{Energy efficiency}

It is well known that both the digital and analog communication schemes can retain the optimal power-distortion trade-off when a i.i.d. Gaussian data transmitted over an additive white Gaussian noise (AWGN) channel $[58,59]$. Also, for the estimation of a Gaus- 
sian source in a decentralized WSN, it is shown in $[21,25,26]$ that the analog schemes outperform (or are as good as) the digital schemes and have the optimal asymptotic scaling behavior using a coherent Gaussian multiple access communication. However, if the rate of generating the symbols at source is slower or faster than the channel symbol rate then analog is no longer optimal, and in fact analog is highly suboptimal. In contrast, digital transmission can retain the optimal power-distortion trade-off for any set of source and channel rates.

To illustrate this concept, let's consider a simple example where a transmitter wishes to transmit a constant $x \in[0,1]$ to a receiver over a AWGN channel, given by

$$
r=y+n
$$

where $y$ and $r$ are the transmitted and received signal, respectively, and $n$ is additive Gaussian noise with zero mean and variance $\sigma^{2}$. Notice that in this example, the rate of generating the symbols at source is slower than the channel symbol rate. Transmitter has two main options to transmit constant $x$ to the receiver : analog or digital.

We first consider the analog approach where transmitter transmits $y=a x$, where $a$ is the amplification factor. In this case, the communication energy is equal to $E_{c o m}=(a x)^{2}$. If the receiver knows the amplification factor, it can estimates the transmitted symbol by

$$
\hat{x}=\frac{r}{a}
$$

where $\hat{x}$ is the estimated value. Here, the mean squared error is

$$
\mathbb{E}\left[(\hat{x}-x)^{2}\right]=\frac{\sigma^{2}}{a^{2}}=\frac{\sigma^{2} x^{2}}{E_{\text {com }}} .
$$


The channel noise variance $\sigma^{2}$ and constant $x$ are fixed. Therefore, the communication energy to obtain an estimate in $\epsilon$-neighborhood of actual value $x$ in mean squared sense is inversely proportional to $\epsilon$. Notice that the trade-off between communication energy and mean squared error in this example is similar to the trade-off derived in chapter 2 for distributed optimization using the analog framework.

We then consider the digital approach. Transmitter first quantizes the constant $x$ into $R$ bits and use digital modulation for communication. In this example, we select the uniform quantization scheme for quantization and 4-QAM digital modulation for communication. Receiver demodulates the received signal to detect the transmitted bits and reconstructs its estimate $\hat{x}$. In this case, the error $\hat{x}-x$ is equal to

$$
\hat{x}-x=e_{Q}+e_{c o m}
$$

where $e_{Q}$ is quantization noise and $e_{\text {com }}$ is the communication error due to channel noise. For the uniform quantization scheme, the power of quantization noise $\mathbb{E}\left[e_{Q}^{2}\right]$ is upper-bounded by

$$
\mathbb{E}\left[e_{Q}^{2}\right] \leq 2^{-2 R}
$$

where $R$ is resolution of quantizer. The power of communication error $\mathbb{E}\left[e_{\text {com }}^{2}\right]$ is upperbounded by

$$
\mathbb{E}\left[e_{\text {com }}^{2}\right] \leq \sum_{i=0}^{R} P_{b} 2^{-2 i} \leq \frac{4 P_{b}}{3},
$$

where $P_{b}$ is bit error probability of channel. Since the communication noise and quantization noise are uncorrelated, the mean squared error is equal to

$$
\mathbb{E}\left[(\hat{x}-x)^{2}\right]=\mathbb{E}\left[e_{Q}^{2}\right]+\mathbb{E}\left[e_{c o m}^{2}\right] \leq 2^{-2 R}+\frac{4 P_{b}}{3}
$$


Here, we select a resolution $R=0.5 \log P_{b}^{-1}$. Therefore, the mean squared error is upper-bounded by

$$
\mathbb{E}\left[(\hat{x}-x)^{2}\right] \leq \frac{7}{3 P_{b}}
$$

Recall that the communication energy required for transmission a message is proportional to the number of bits in the message if M-QAM modulation is used [60]. Therefore, the communication energy for 4-QAM is given as

$$
E_{\text {com }}=w \log \left(\frac{1}{P_{b}}\right) R
$$

where $w$ is a constant value depending on the power spectrum density of channel noise and the the number of bits per M-QAM symbol, as it is mentioned in chapter 3. Replace resolution $R$ to obtain

$$
E_{\text {com }}=\frac{w}{2} \log ^{2} \frac{1}{P_{b}}
$$

It follows from (4.1) and (4.2) that the communication energy required to obtain an estimate in $\epsilon$-neighborhood of actual value $x$ in mean square sense is proportional to $\log ^{2} 1 / \epsilon$. This example clearly shows that digital communication scheme outperforms the analog communication scheme when the the rate of generating the symbols at source is slower than the channel symbol rate. Notice that the trade-off between communication energy and mean squared error in this example is similar to the trade-off that derived in chapter 3 for distributed optimization using the digital framework. 


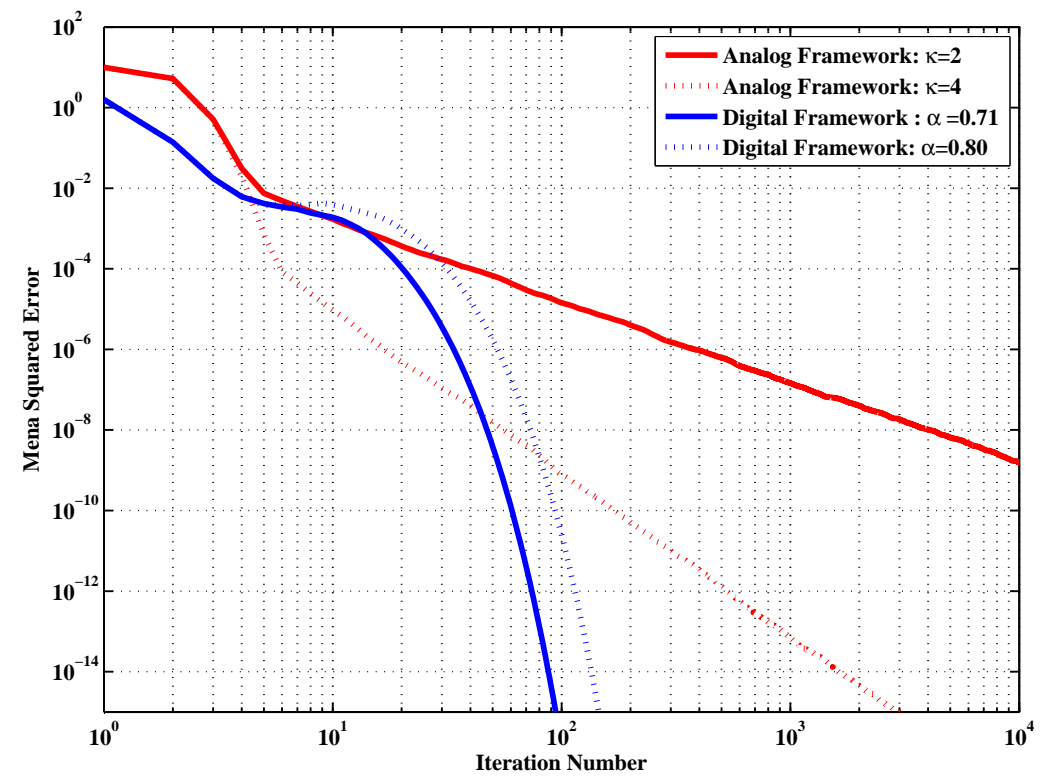

Figure 4.1: Mean squared error versus iteration number

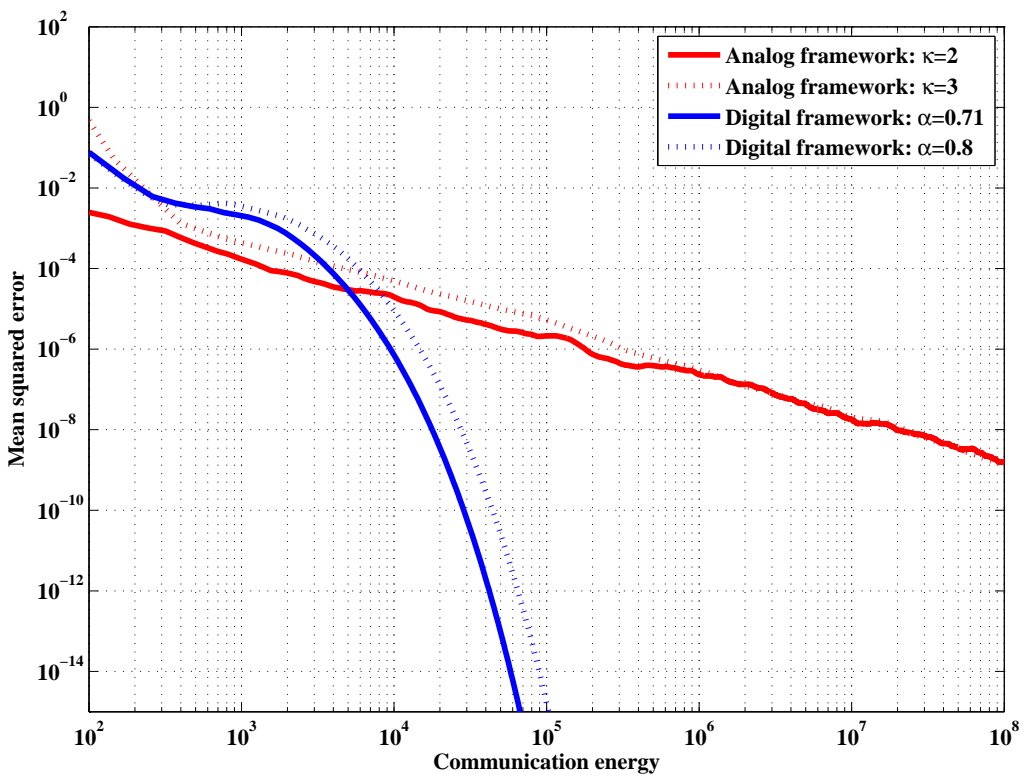

Figure 4.2: Mean squared error versus communication energy 


\subsection{Simulation results}

In this section, we consider a simple example to compare the trade-off between communication energy and mean squared error in analog and digital frameworks. Consider a network of 10 nodes which wish to minimize a quadratic convex function

$$
f(\mathbf{x})=\frac{1}{2} \mathbf{x}^{T} \mathbf{A} \mathbf{x}+\mathbf{b}^{T} \mathbf{x}+c .
$$

We generate a symmetric positive definite random matrix $\mathbf{A}$ such that $f(\mathbf{x}) \in \mathcal{F}_{2,1}$, and select vector $\mathbf{b}$ such that $f(\mathbf{x})$ has a minimum point at $\mathbf{x}=[0.5, \ldots, 0.5]^{T}$. The communication channels are additive white gaussian channels, and the channel noise variances $\sigma_{\ell, j}^{2}$ are taken to be proportional to $d_{\ell, j}^{2}$ where $d_{\ell, j}$ is the distance between node $S_{\ell}$ and $S_{j}$. The mean squared error for both analog and digital frameworks averaged over 10 runs are shown in Figure 4.1. This figure confirms that the convergence rate of analog algorithm is sub-linear $\left(\mathcal{O}\left(t^{-\kappa}\right)\right)$ which is less than the convergence rate of the digital algorithm $\mathcal{O}\left(\alpha^{t}\right)$. Notice that the choice of $\alpha$ should satisfy (3.9). In particular, $\alpha \geq \sqrt{1-1 / M}=\sqrt{2} / 2$. Figure 4.2 shows the communication energy required to obtain $\epsilon$-minimizer. As expected, in the analog framework, the mean squared error decreases sub-linearly with communication energy independent of choice of $\kappa$. In the digital framework, the mean squared error decreases exponentially with communication energy. However, this does not mean that the digital framework outperforms the analog framework in terms of energy for any value of mean squared error. For example, in our simulation, the analog framework requires less energy to obtain an $\epsilon$-minimizer of $f(\mathbf{x})$ compared with digital framework for $\epsilon \geq 10^{-4}$ (Figure 4.2). For value $\epsilon \leq 10^{-6}$, the digital framework consumes less energy than the analog framework. 


\section{Chapter 5}

\section{Conclusion and Future Work}

The goal of this dissertation was to establish the relation between the communication energy and the convergence condition and rate in distributed optimization problems. In this chapter, as this dissertation comes to the end, we summarize our contributions and provide directions for future research which can complement this study.

\subsection{Conclusion}

We studied the problem of distributed optimization of strongly convex functions in an energy-constrained network. We considered both analog and digital communication schemes. For the analog communication scheme, we showed that the communication energy to obtain an $\epsilon$-minimizer of a function in the mean square sense must grow at least at the rate of $\Omega(1 / \epsilon)$. We derived an isotropic universal design which attains the minimum energy bound within a constant factor. For digital communication schemes, we introduced a distributed algorithm which requires $\mathcal{O}\left(\log ^{2} 1 / \epsilon\right)$ communication en- 
ergy to obtain an $\epsilon$-minimizer of strongly convex cost functions.

\subsection{Directions for future work}

For future work, there are several directions to improve and extend the results presented in this thesis. One direction to persuade is to consider different coding strategies for distributed optimization problem to reduce the communication cost. It was proved in [61] that any distributed algorithm which runs in time $T$ on two nodes with noiseless communication channels can be simulated with noisy communication channels (each a binary symmetric channel of capacity $C$ ) in time proportional to $T / C$, and the probability of failure of the algorithm is $e^{\Omega(T)}$. This result was extended to the multiple node case in [62]. However, the computational overhead for the decoding process is increasing exponentially with the number of iterations. In addition, only a probabilistic argument is given for the existence of the tree code without giving any effective construction algorithm. Recently, an improvement of the randomized construction for the tree code is presented in [63]. In [54], a distributed algorithm for a network of two nodes is proposed that requires $\mathcal{O}(\log 1 / \epsilon)$ iterations with constant number of transmitted bits in each iteration to obtain an $\epsilon$-minimizer of a strongly convex function, assuming distortionless communication channels. The results in [54] and [61] indicate that theoretically $\Omega(\log 1 / \epsilon)$ number of transmitted bits is sufficient to obtain an $\epsilon$-minimizer of strongly convex cost functions. Notice that the communication energy is linearly proportional to the number of transmitted bit in the noisy communication channel provided that the probability of error and digital modulation scheme are given. In other words, the re-

quired communication energy to obtain an $\epsilon$-minimizer theoretically grows at the rate of $\mathcal{O}(\log 1 / \epsilon)$ using the digital communication scheme and proper coding scheme. 
This thesis studied the communication energy for distributed optimization of a strongly convex function. A natural extension of this work is to consider a more general cost function as it was considered in [54] for distortion-less communication channels in a network of two nodes. It is also interesting to extend the results of this work to the different distributed optimization set-ups such as minimization of a sum of functions, when each component function is available only to a specific node. The optimization problem of this kind arise in sensor networks [64] and a network of database where the privacy considerations may not allow the sharing of the objective functions [65]. Recently, authors in [66] studied this problem assuming each node knows the sub-gradient of its corresponding function with stochastic errors and proposed an algorithm based on the sub-gradient incremental algorithms which converge in mean square sense to the optimal point if error in the sub-gradient evaluation satisfies some condition. However, no attempt is made to derive the impact of communication cost on the convergence of algorithm.

In this thesis, we did not consider asynchronous node operations. There are two major methods to address this issue: Timing synchronization among nodes and distributed asynchronous algorithms. Timing synchronization in a large WSN is difficult (if not impossible). Estimating the clock timing of neighboring nodes or the global clock of all nodes can be done via exchanging time-stamps in various network-layer timing protocols such as level-based synchronization $[67,68]$ and the reference broadcast synchronization [69]. All these protocols assume that the receive-time is perfectly available via physical layer synchronization. However, this is not a realistic assumption in a WSN. In a distributed asynchronous algorithm, nodes are allowed to perform their local tasks at their own pace even when the current data of some other nodes are not available. 
Furthermore, nodes are not required to communicate their results after each iteration. This feature can potentially preserve the energy of nodes with lower battery power in a WSN. The convergence issues of distributed asynchronous algorithms are well studied in literatures such as $[52,53,70]$. However, more work should be done to determine the trade-off between the energy and convergence in these algorithms. 


\section{Bibliography}

[1] A. Hasler, I. Talzi, J. Beutel, C. Tschudin, and S. Gruber, "Wireless sensor networks in permafrost research concept, requirements, implementation and challenges," in Proceedings of the 9th International Conference on Permafrost, 2008, pp. 669-674.

[2] A. Elsaify, P. Padhy, K. Martinez, and G. Zou, "Gwmac- a tdma based mac protocol for a glacial sensor network," in Proceedings of 4th ACM PE-WASUN, 2007.

[3] C. o. E. National Research Council (U.S.) Committee on Alternative Technologies to Replace Antipersonnel Landmines. National Research Council (U.S.) and O. o. I. A. N. A. o. S. U. Technical Systems. National Research Council (U.S.), Alternative technologies to replace antipersonnel landmines. National Academies Press, 2001.

[4] IEEE Standard for a Smart Transducer Interface for Sensors and Actuators - Common Functions, Communication Protocols, and Transducer Electronic Data Sheet (TEDS) Formats. IEEE Press, 2007. 
[5] L. Xiao, S. Boyd, and S.-J. Kim, "Distributed average consensus with least-meansquare deviation," Journal of Optimization Theory and Applications, vol. 67, no. 1, pp. 33-46, 2007.

[6] R. Olfati-Saber and R. M. Murray, "Consensus problems in networks of agents with switching topologies and time-delays," IEEE Transactions on Automatic Control, vol. 49, no. 9, pp. 1520-1533, 2004.

[7] L. Xiao and S. Boyd, "Fast linear iterations for distributed averaging," Systems and Control Letters, vol. 53, pp. 65-78, 2004.

[8] L. Xiao, S. Boyd, and S. Lall, "A scheme for robust distributed sensor fusion based on average consensus," in Proceedings of the 4th international symposium on Information Processing in Sensor Networks. IEEE Press, 2005, pp. 63-70.

[9] V. Delouille, R. Neelamani, and R. Baraniuk, "Robust distributed estimation in sensor networks using the embedded polygons algorithm," in Proceedings of the 3rd International Symposium on Information Processing in Sensor Networks, April 2004, pp. 405-413.

[10] D. Scherber and H. Papadopoulos, "Distributed computation of averages over ad hoc networks," IEEE Journal on Selected Areas in Communications, vol. 23, no. 4, pp. 776-787, April 2005.

[11] I. D. Schizas, A. Ribeiro, and G. B. Giannakis, "Consensus in ad hoc wsns with noisy links - part i: Distributed estimation of deterministic signals," IEEE Transactions on Signal Processing, vol. 56, no. 1, pp. 350-364, 2008. 
[12] M. W. Carter, H. H. Jin, M. A. Saunders, and Y. Ye, "Spaseloc: An adaptive subproblem algorithm for scalable wireless sensor network localization," SIAM Journal on Optimization, vol. 17, no. 4, pp. 1102-1128, 2006.

[13] Shockfish, TinyNode 584 Users Manual.

[14] J. Speyer, "Computation and transmission requirements for a decentralized linearquadratic-gaussian control problem," IEEE Transactions on Automatic Control, vol. 24, no. 2, pp. 266-269, April 1979.

[15] D. Castanon and D. Teneketzis, "Distributed estimation algorithms for nonlinear systems," IEEE Transactions on Automatic Control, vol. 30, no. 5, pp. 418-425, May 1985.

[16] A. Willsky, M. Bello, D. Castanon, B. Levy, and G. Verghese, “Combining and updating of local estimates and regional maps along sets of one-dimensional tracks," IEEE Transactions on Automatic Control, vol. 27, no. 4, pp. 799-813, August 1982.

[17] Z. Chair and P. Varshney, "Distributed bayesian hypothesis testing with distributed data fusion," IEEE Transactions on Systems, Man and Cybernetics, vol. 18, no. 5, pp. 695-699, September/October 1988.

[18] Z.-Q. Luo and J. Tsitsiklis, "Data fusion with minimal communication," IEEE Transactions on Information Theory, vol. 40, no. 5, pp. 1551-1563, September 1994. 
[19] M. Abdallah and H. Papadopoulos, "Sequential signal encoding and estimation for distributed sensor networks," in Proceedings of IEEE International Conference on Acoustics, Speech, and Signal Processing, 2001, pp. 2577-2580.

[20] H. Papadopoulos, G. Wornell, and A. Oppenheim, "Sequential signal encoding from noisy measurements using quantizers with dynamic bias control," IEEE Transactions on Information Theory, vol. 47, no. 3, pp. 978-1002, March 2001.

[21] M. Gastpar and M. Vetterli, "Source-channel communication in sensor networks," Lecture Notes in Computer Science, vol. 2634, pp. 162-177, April 2003.

[22] M. Gastpar, "Uncoded transmission is exactly optimal for a simple gaussian "sensor" network," in Proceedings of Information Theory and Applications Workshop, 29 January-February 2 2007, pp. 177-182.

[23] A. D. Sarwate and M. Gastpar, "Fading observation alignment via feedback," in Proceedings of the 4th international symposium on Information processing in sensor networks. Piscataway, NJ, USA: IEEE Press, 2005, p. 42.

[24] K. Eswaran and M. Gastpar, "On the quadratic awgn ceo problem and nongaussian sources," in Proceedings of International Symposium on Information Theory, September 2005, pp. 219-223.

[25] K. Liu, H. El Gamal, and A. Sayeed, "On optimal parametric field estimation in sensor networks," in Proceedings of the 13th IEEE/SP Workshop on Statistical Signal Processing, July 2005, pp. 1170-1175.

[26] W. Bajwa, A. Sayeed, and R. Nowak, "Matched source-channel communication for field estimation in wireless sensor network," in Proceedings of the 4th Interna- 
tional Symposium on Information Processing in Sensor Networks, April 2005, pp. 332-339.

[27] G. Mergen, V. Naware, and L. Tong, "symptotic detection performance of typebased multiple access in sensor networks," in Proceedings of 6th IEEE International Workshop on Signal Processing Advances in Wireless Communications, June 2005, pp. 1018-1022.

[28] G. Mergen and L. Tong, "Type based estimation over multiaccess channels," IEEE Transactions on Signal Processing, vol. 54, no. 2, pp. 613-626, February 2006.

[29] K. Liu and A. M. Sayeed, "Optimal distributed detection strategies for wireless sensor networks," in Proceedings of 42nd Annual Allerton Conference on Communications, Control and Computing, October 2004.

[30] D. Marco, E. Duarte-Melo, M. Liu, and D. Neuhoff, "The many-to-one transport capacity of a dense wireless sensor network and the compressibility of its data," pp. 1-16, April 2003.

[31] P. Ishwar, R. Puri, K. Ramchandran, and S. Pradhan, "On rate-constrained distributed estimation in unreliable sensor networks," IEEE Journal on Selected Areas in Communications, vol. 23, no. 4, pp. 765-775, April 2005.

[32] J.-J. Xiao, S. Cui, Z.-Q. Luo, and A. Goldsmith, "Linear coherent decentralized estimation," IEEE Transactions on Signal Processing, vol. 56, no. 2, pp. 757-770, February 2008. 
[33] enbin Guo, J.-J. Xiao, and S. Cui, "An efficient water-filling solution for linear coherent joint estimation," IEEE Transactions on Signal Processing, vol. 56, no. 10, pp. 5301-5305, October 2008.

[34] Z.-Q. Luo, "An isotropic universal decentralized estimation scheme for a bandwidth constrained ad hoc sensor network," IEEE Journal on Selected Areas in Communications, vol. 23, no. 4, pp. 735-744, April 2005.

[35] Z.-Q. Luo and J.-J. Xiao, "Decentralized estimation in an inhomogeneous environment," in Proceedings of International Symposium on Information Theory, June-2 July 2004, pp. 520-.

[36] K. Zhang, X. Li, P. Zhang, and H. Li, "Optimal linear estimation fusion - part vi: sensor data compression," in Proceedings of the 6th International Conference of Information Fusion, vol. 1, 2003, pp. 221-228.

[37] Z.-Q. Luo, G. Giannakis, and S. Zhang, "Optimal linear decentralized estimation in a bandwidth constrained sensor network," in Proceedings of International Symposium on Information Theory, September 2005, pp. 1441-1445.

[38] I. Schizas, G. Giannakis, and Z.-Q. Luo, "Distributed estimation using reduceddimensionality sensor observations," IEEE Transactions on Signal Processing, vol. 55, no. 8, pp. 4284-4299, August 2007.

[39] S. Cui, J.-J. Xiao, A. Goldsmith, Z.-Q. Luo, and H. Poor, "Estimation diversity and energy efficiency in distributed sensing," IEEE Transactions on Signal Processing, vol. 55, no. 9, pp. 4683-4695, September 2007. 
[40] J.-J. Xiao, S. Cui, Z.-Q. Luo, and A. Goldsmith, "Power scheduling of universal decentralized estimation in sensor networks," IEEE Transactions on Signal Processing, vol. 54, no. 2, pp. 413-422, February 2006.

[41] R. Olfati-Saber and J. Shamma, "Consensus filters for sensor networks and distributed sensor fusion," in Proceedings of the 44th IEEE Conference on Decision and Control, and European Control Conference, Dec. 2005, pp. 6698-6703.

[42] D. P. Spanos, R. Olfati-Saber, and R. J. Murray, "Distributed sensor fusion using dynamic consensus," in Proceedings of the 16th IFAC World Congress, July 2005, pp. 6698-6703.

[43] A. Speranzon, C. Fischione, K. Johansson, and A. Sangiovanni-Vincentelli, “A distributed minimum variance estimator for sensor networks," IEEE Journal on Selected Areas in Communications, vol. 26, no. 4, pp. 609-621, May 2008.

[44] R. Carli, A. Chiuso, L. Schenato, and S. Zampieri, "Distributed kalman filtering based on consensus strategies," IEEE Journal on Selected Areas in Communications, vol. 26, no. 4, pp. 622-633, May 2008.

[45] S. Barbarossa and G. Scutari, "Decentralized maximum-likelihood estimation for sensor networks composed of nonlinearly coupled dynamical systems," IEEE Transactions on Signal Processing, vol. 55, no. 7, pp. 3456-3470, July 2007.

[46] L. Prescosolido, S. Barbarossa, and G. Scutari, "Decentralized detection and localization through sensor networks designed as a population of selfsynchronized oscillators," in Proceedings of IEEE International Conference on Acoustics, Speech and Signal Processing, May 2006, p. 981984. 
[47] M. G. Rabbat, R. D. Nowak, and J. A. Bucklew, "Generalized consensus algorithms in networked systems with erasure links," in Proceedings of 6th IEEE Workshop on Signal Processing Advances in Wireless Communications, 2005, pp. 10881092.

[48] Y. Rabani, A. Sinclair, and R. Wanka, "Local divergence of markov chains and the analysis of iterative load-balancing schemes," in Proceedings of the 39th IEEE Symposium on Foundations of Computer Science (FOCS 98, November 1998, pp. 694-703.

[49] M. E. Yildiz and A. Scaglione, "Differential nested lattice encoding for consensus problems," in Proceedings of the 6th international conference on Information processing in sensor networks. New York, NY, USA: ACM, 2007, pp. 89-98.

[50] A. Kashyap, T. Basar, and R. Srikant, "Quantized consensus," in Proceedings of IEEE International Symposium on Information Theory, July 2006, pp. 635-639.

[51] T. C. Aysal, M. J. Coates, and M. G. Rabbat, "Distributed average consensus using probabilistic quantization," in Proceedings of 14th IEEE/SP Workshop on Statistical Signal Processing, August 2007, pp. 640-644.

[52] J. N. Tsitsiklis, D. Bertsekas, and M. Athans, "Distributed asynchronous deterministic and stochastic gradient optimization algorithms," IEEE Transactions on Automatic Control, vol. 31, no. 9, pp. 803-812, 1986.

[53] D. Bertsekas and J. N. Tsitsiklis, Parallel and Distributed Computation Numerical Methods. Englewood Cliffs, NJ: Prentice-Hall, 1989. 
[54] J. N. Tsitsiklis and Z. Luo, "Communication complexity of convex optimization," Journal of Complexity, vol. 3, no. 3, pp. 231-243, 1987.

[55] H. Robbins and S. Monro, "A stochastic approximation method," Annals of Mathematical Statistics, vol. 22, no. 3, pp. 400-407, 1951.

[56] D. P. Bertsekas and J. N. Tsitsiklis, "Gradient convergence in gradient methods with errors," SIAM Journal on Optimization, vol. 10, no. 3, pp. 627-642, 2000.

[57] J. G. Prozakis, Digital communication, 4th ed. Englewood Cliffs, NJ: PrenticeHall, 2000.

[58] T. J. Goblick, "Theoretical limitations on the transmission of data from analog sources," IEEE Transactions on Information Theory, vol. 11, no. 4, pp. 558-567, Oct 1965.

[59] M. Gastpar, B. Rimoldi, and M. Vetterli, "To code, or not to code: lossy sourcechannel communication revisited," IEEE Transactions on Information Theory, vol. 49, no. 5, pp. 1147-1158, May 2003.

[60] S. Cui, A. Goldsmith, and A. Bahai, "Energy-constrained modulation optimization," IEEE Transactions on Wireless Communications, vol. 4, no. 5, pp. 2349 2360, 2005.

[61] L. J. Schulman, "Deterministic coding for interactive communication," in Proceedings of the 25th annual ACM symposium on Theory of computing, 1993, pp. $747-756$. 
[62] S. Rajagopalan and L. J. Schulman, "A coding theorem for distributed computation," in Proceedings of the 26th annual ACM symposium on Theory of computing, 1994, pp. 790-799.

[63] M. Peczarski, "An improvement of the tree code construction," Information Processing Letters, vol. 99, no. 3, pp. 92-95, 2006.

[64] M. Rabbat and R. Nowak, "Quantized incremental algorithms for distributed optimization," IEEE Journal on Selected Areas in Communications, vol. 23, no. 4, pp. 798-808, April 2005.

[65] B. Park and H. Kargupta, Data Mining Handbook. Lawrence Erlbaum Associates, 2002, ch. Distributed Data Mining: Algorithms, Systems, and Applications.

[66] S. S. Ram, A. Nedich, and V. V. Veeravalli, "Distributed stochastic subgradient projection algorithms for convex optimization," 2008. [Online]. Available: http://www.citebase.org/abstract?id=oai:arXiv.org:0811.2595

[67] S. Ganeriwal, R. Kumar, and M. B. Srivastava, "Timing-sync protocol for sensor networks," in Proceedings of the 1st international conference on Embedded networked sensor systems. New York, NY, USA: ACM, 2003, pp. 138-149.

[68] J. V. Greunen and J. Rabaey, "Lightweight time synchronization for sensor networks," in Proceedings of the 2nd ACM international conference on Wireless sensor networks and applications. New York, NY, USA: ACM, 2003, pp. 11-19.

[69] J. Elson, L. Girod, and D. Estrin, "Fine-grained network time synchronization using reference broadcasts," ACM SIGOPS Operating Systems Review, vol. 36, no. SI, pp. 147-163, 2002. 
[70] Z.-Q. Luo and P. Tseng, "On the rate of convergence of a distributed asynchronous routing algorithm," IEEE Transactions on Automatic Control, vol. 39, no. 5, pp. 1123-1129, May 1994.

[71] A. S. Nemirovsky and D. B. Yudin, Problem Convexity and Method efficiency in Optimization. New York: Wiley, 1983. 


\section{Appendix A}

\section{Proof of (2.11)-(2.12)}

We first compute the upper bound on $\left\|\mathbf{e}_{i n}(t)\right\|^{2}$.

$$
\left\|\mathbf{e}_{i n}(t)\right\|^{2} \leq\left\|\prod_{i=1}^{t}\left(I-\frac{\mathbf{A}}{i^{\beta}}\right)\right\|^{2}\|\mathbf{e}(0)\|^{2} .
$$

Corollary G.1 in Appendix G gives rise to (2.11).

We then compute $\mathbb{E}\left[\left\|\mathbf{e}_{c h}(t)\right\|^{2}\right]$. Consider the eigenvalue decomposition of the matrix A:

$$
\Lambda \triangleq \mathbf{P}^{T} \mathbf{A P}=\operatorname{diag}\left(\lambda_{1}, \ldots, \lambda_{n}\right)
$$

where $\lambda_{\ell}$ are eigenvalues of the matrix $\mathbf{A}$, and matrix $\mathbf{P}$ is orthonormal. Define $p_{m, q}$ as the $(m, q)$-th element of the matrix $\mathbf{P}$, and $v_{m}(i)$ as $m$-th element of vector $\mathbf{v}(i)$. From 
the definition of $\mathbf{e}_{c h}(t)$, equation (2.10), we have

$$
\mathbb{E}\left[\left\|\mathbf{e}_{c h}(t)\right\|^{2}\right]=\mathbb{E}\left[\left\|\sum_{j=1}^{t}\left[\begin{array}{c}
\sum_{m=1}^{n} b_{1}(j, t) p_{m, 1} v_{m}(j-1) \\
\vdots \\
\sum_{m=1}^{n} b_{n}(j, t) p_{m, n} v_{m}(j-1)
\end{array}\right]\right\|^{2}\right]
$$

where

$$
b_{q}(j, t)=\frac{1}{j^{\beta}} \prod_{i=j+1}^{t}\left(1-\frac{\lambda_{q}}{i^{\beta}}\right) .
$$

Since the channel noise is spatially uncorrelated and has zero mean, $v_{m}(j-1), m \in$ $\{1, \ldots, n\}$ are also uncorrelated zero mean random variables. Hence,

$$
\mathbb{E}\left[\left\|\mathbf{e}_{c h}(t)\right\|^{2}\right]=\sum_{m, q=1}^{n} p_{m, q}^{2} \mathbb{E}\left[\left(\sum_{j=1}^{t} b_{q}(j, t) v_{m}(j-1)\right)^{2}\right]
$$

Replace $v_{m}(j-1)$ in inner sum in (A.1) with (2.7), to obtain

$$
\begin{aligned}
\mathbb{E}\left[\left\|\mathbf{e}_{c h}(t)\right\|^{2}\right] & =\sum_{m, q=1}^{n} p_{m, q}^{2} \mathbb{E}\left[\left(\sum_{j=1}^{t} b_{q}(j, t) \sum_{\ell=1}^{n} a_{m, \ell} \sum_{i=1}^{j-1} \prod_{k=i+1}^{j-1} \gamma_{\ell}(k) \frac{n_{\ell, m}(i)}{\psi_{\ell}(i)}\right)^{2}\right] \\
& =\sum_{m, q, \ell=1}^{n} p_{m, q}^{2} a_{m, \ell}^{2} \mathbb{E}\left[\left(\sum_{j=1}^{t} b_{q}(j, t) \sum_{i=1}^{j-1} \prod_{k=i+1}^{j-1} \gamma_{\ell}(k) \frac{n_{\ell, m}(i)}{\psi_{\ell}(i)}\right)^{2}\right] \\
& =\sum_{m, q, \ell=1}^{n} p_{m, q}^{2} a_{m, \ell}^{2} \sigma_{\ell, m}^{2} \sum_{i=1}^{t-1}\left(\frac{\alpha_{\ell}(i, t, q)}{\psi_{\ell}(i)}\right)^{2}
\end{aligned}
$$


where $\sigma_{\ell, m}^{2}$ is the variance of channel noise between node $S_{\ell}$ and node $S_{m}, n_{\ell, m}(i)$, and

$$
\alpha_{\ell}(i, t, q)=\sum_{j=i+1}^{t} \frac{1}{j^{\beta}} \prod_{k=j+1}^{t}\left(1-\frac{\lambda_{q}}{k^{\beta}}\right) \prod_{k=i+1}^{j-1} \gamma_{\ell}(k) .
$$

In equation (A.2), the last two steps are due to the fact that the noises $n_{\ell, m}(i), \ell, i \in$ $\{1, \ldots, t\}$, are uncorrelated. 


\section{Appendix B}

\section{Proof of Theorem 1}

Define vector $\mathbf{y}(i) \triangleq\left[y_{1}(i), \ldots, y_{n}(i)\right]^{T}$ as the vector of signals transmitted by the nodes at iteration $i ; \boldsymbol{\Gamma}(i) \triangleq \operatorname{diag}\left(\gamma_{1}(i), \ldots, \gamma_{n}(i)\right)$, and $\boldsymbol{\Psi}(i) \triangleq \operatorname{diag}\left(\psi_{1}(i), \ldots, \psi_{n}(i)\right)$. The total communication energy is

$$
\begin{aligned}
E_{\text {com }}(t) & =\sum_{i=1}^{t-1} \mathbb{E}\|\mathbf{y}(i)\|^{2} \\
& =\sum_{i=1}^{t-1} \mathbb{E}\|\mathbf{\Psi}(i)[\mathbf{x}(i)-\boldsymbol{\Gamma}(i) \mathbf{x}(i-1)]\|^{2} \\
& =\sum_{i=1}^{t-1} \mathbb{E}\left\|\mathbf{\Psi}(i)\left[\left(\mathbf{I}-\mathbf{\Gamma}(i)-\frac{\mathbf{A}}{i^{\beta}}\right) \mathbf{x}(i-1)+\frac{\mathbf{A} \mathbf{x}^{*}-\mathbf{v}(i-1)}{i^{\beta}}\right]\right\|^{2},
\end{aligned}
$$

where the last equality is due to the iteration update of $\mathbf{x}(i)$ in (2.6), and the fact that $\mathbf{b}=-\mathbf{A} \mathbf{x}^{*}$. The vector $\mathbf{x}(i-1)$ in (B.2) is expressed by

$$
\begin{aligned}
\mathbf{x}(i-1) & =\left(\mathbf{x}(i-1)-\mathbf{x}^{*}\right)+\mathbf{x}^{*}=\mathbf{e}(i-1)+\mathbf{x}^{*} \\
& =\mathbf{e}_{i n}(i-1)+\mathbf{e}_{c h}(i-1)+\mathbf{x}^{*}
\end{aligned}
$$


where $\mathbf{e}_{i n}(t)$ and $\mathbf{e}_{c h}(t)$ represent the errors due to initialization and channel noise, respectively. Substituting the value of $\mathbf{x}(i-1)$ into the total communication energy in (B.2), and using the fact that the channel noise is zero mean, and independent of initial point and optimal point, we obtain

$$
E_{c o m}(t)=E_{i n}(t)+E_{c h}(t)
$$

where

$$
\begin{aligned}
& E_{i n}(t) \triangleq \sum_{i=1}^{t-1}\left\|\boldsymbol{\Psi}(i)\left[(\mathbf{I}-\boldsymbol{\Gamma}(i)) \mathbf{x}^{*}+\left(\mathbf{I}-\boldsymbol{\Gamma}(i)-\frac{\mathbf{A}}{i^{\beta}}\right) \mathbf{e}_{i n}(i-1)\right]\right\|^{2}, \\
& E_{c h}(t) \triangleq \sum_{i=1}^{t-1} \mathbb{E}\left\|\boldsymbol{\Psi}(i)\left[\left(\mathbf{I}-\boldsymbol{\Gamma}(i)-\frac{\mathbf{A}}{i^{\beta}}\right) \mathbf{e}_{c h}(i-1)+\frac{\mathbf{v}(i-1)}{i^{\beta}}\right]\right\|^{2} .
\end{aligned}
$$

Here, $E_{i n}(t)$ is the communication energy in the noise-free scenario, and $E_{c h}(t)$ is the energy caused by the presence of channel noise. Notice that the communication energy is lower bounded by

$$
E_{\text {com }}(t) \geq E_{\text {in }}(t)=\sum_{\ell=1}^{n} E_{\text {in, } \ell}(t)
$$

In equation (B.5), $E_{i n, \ell}(t)$ is the amount of energy spent by node $S_{\ell}$ in the noise-free scenario which is given by

$$
E_{i n, \ell}(t)=\sum_{i=1}^{t-1} \psi_{\ell}^{2}(i) d_{\ell}^{2}(i)
$$


where

$$
\mathbf{d}(i) \triangleq(\mathbf{I}-\boldsymbol{\Gamma}(i)) \mathbf{x}^{*}+\left(\mathbf{I}-\boldsymbol{\Gamma}(i)-\frac{\mathbf{A}}{i^{\beta}}\right) \mathbf{e}_{i n}(i-1)
$$

Recall that $\mathbb{E}\left[\|\mathbf{e}(t)\|^{2}\right] \geq \mathbb{E}\left[\left\|\mathbf{e}_{c h}(t)\right\|^{2}\right]$, we have

$$
\begin{aligned}
\mathbb{E}\left[\|\mathbf{e}(t)\|^{2}\right] E_{c o m}(t) & \geq \mathbb{E}\left[\left\|\mathbf{e}_{c h}(t)\right\|^{2}\right] E_{i n}(t) \\
& =\sum_{\ell=1}^{n} \mathbb{E}\left[e_{c h, \ell}^{2}(t)\right] \sum_{\ell=1}^{n} E_{i n, \ell}(t) \\
& \geq \sum_{\ell=1}^{n} \mathbb{E}\left[e_{c h, \ell}^{2}(t)\right] E_{i n, \ell}(t),
\end{aligned}
$$

where $\mathbb{E}\left[e_{c h, \ell}^{2}(t)\right]$, the contribution of channel noise between node $S_{\ell}$ and its neighbors to the mean squared error, is (A.2)

$$
\mathbb{E}\left[e_{c h, \ell}^{2}(t)\right]=\sum_{m, q=1}^{n} p_{m, q}^{2} a_{m, \ell}^{2} \sigma_{\ell, m}^{2} \sum_{i=1}^{t-1}\left(\frac{\alpha_{\ell}(i, t, q)}{\psi_{\ell}(i)}\right)^{2}
$$

Substituting (B.6) and (B.8) into (B.7) and using the Cauchy-Schwartz inequality for each summand, we obtain

$$
\begin{aligned}
\mathbb{E}\left[\left\|\mathbf{e}_{c h}(t)\right\|^{2}\right] \sum_{i=1}^{t-1} \psi_{\ell}^{2}(i) d_{\ell}^{2}(i) & =\left(\sum_{m, q=1}^{n} a_{m, \ell}^{2} p_{m, q}^{2} \sigma_{\ell, m}^{2} \sum_{i=1}^{t-1}\left(\frac{\alpha_{\ell}(i, t, q)}{\psi_{\ell}(i)}\right)^{2}\right) \sum_{i=1}^{t-1} \psi_{\ell}^{2}(i) d_{\ell}^{2}(i) \\
& \geq \sum_{m, q=1}^{n} a_{m, \ell}^{2} p_{m, q}^{2} \sigma_{\ell, m}^{2}\left(\sum_{i=1}^{t-1}\left|\alpha_{\ell}(i, t, q) d_{\ell}(i)\right|\right)^{2}
\end{aligned}
$$

Here, we derive the asymptotic lower bound on the inner sum in (B.9). Define two 
sequences $\{w(i)\}$ and $\{z(i)\}$ such that their sum is equal to $d_{\ell}(i) \alpha_{\ell}(i, t, q)$ :

$$
\begin{aligned}
& w(i) \triangleq\left(1-\gamma_{\ell}(i)\right) \alpha_{\ell}(i, t, q)\left(x_{\ell}^{*}+e_{i n, \ell}(i-1)\right), \\
& z(i) \triangleq \frac{\alpha_{\ell}(i, t, q)}{i^{\beta}}\left\langle[\mathbf{A}]_{\ell}, \mathbf{e}_{i n}(i-1)\right\rangle
\end{aligned}
$$

where $[\mathbf{A}]_{\ell}$ is the $\ell$-th row of the matrix $\mathbf{A}$, and $x_{\ell}^{*}$ and $e_{i n, \ell}(t)$ are the $\ell$-th elements of the vector $\mathbf{x}^{*}$ and $\mathbf{e}_{\ell}(t)$, respectively. For $1 \leq t_{\ell} \leq t-1$, we have

$$
\begin{aligned}
\sum_{i=1}^{t-1}\left|\alpha_{\ell}(i, t, q) d_{\ell}(i)\right| & \geq \sum_{i=t_{\ell}}^{t-1}\left|\alpha_{\ell}(i, t, q) d_{\ell}(i)\right| \\
& =\sum_{i=t_{\ell}}^{t-1}|w(i)+z(i)| \\
& \geq \sum_{i=t_{\ell}}^{t-1}|w(i)|-\sum_{i=t_{\ell}}^{t-1}|z(i)|,
\end{aligned}
$$

where the last stem is due to the triangle inequality. Therefore,

$$
\lim _{t \rightarrow \infty} \sum_{i=1}^{t-1}\left|\alpha_{\ell}(i, t, q) d_{\ell}(i)\right| \geq \lim _{t \rightarrow \infty} \sum_{i=t_{\ell}}^{t-1}|w(i)|-\lim _{t \rightarrow \infty} \sum_{i=t_{\ell}}^{t-1}|z(i)|
$$

We then use the following lemma which is proven in Appendix C.

Lemma B.1 For two sequences $\{w(i)\}$ and $\{z(i)\}$ as defined at equations (B.10) (B.11), and positive numbers $\delta_{6}, \delta_{7}$, there exists a large enough $t_{\ell}$ (depending on $\delta_{6}, \delta_{7}$ ) such that

$$
\lim _{t \rightarrow \infty} \sum_{i=t_{\ell}}^{t-1}|w(i)| \geq \frac{\left(1-\delta_{6}\right)\left|x_{\ell}^{*}\right|}{\lambda_{q}}
$$




$$
\lim _{t \rightarrow \infty} \sum_{i=t_{\ell}}^{t-1}|z(i)| \leq \delta_{7}
$$

It follows from Lemma B.1 that

$$
\lim _{t \rightarrow \infty} \sum_{i=1}^{t-1}\left|\alpha_{\ell}(i, t, q) d_{\ell}(i)\right| \geq \frac{\left(1-\delta_{6}\right)\left|x_{\ell}^{*}\right|}{\lambda_{q}}-\delta_{7} .
$$

Define $\delta_{3} \triangleq \delta_{6}+\delta_{7} \lambda_{q} /\left|x_{\ell}^{*}\right|$. We have

$$
\lim _{t \rightarrow \infty} \sum_{i=1}^{t-1}\left|\alpha_{\ell}(i, t, q) d_{\ell}(i)\right| \geq \frac{\left(1-\delta_{3}\right)\left|x_{\ell}^{*}\right|}{\lambda_{q}}
$$

Notice that $\delta_{3}$ can be made arbitrary small, namely $\delta_{3}=1 / 2$. Therefore,

$$
\liminf _{t \rightarrow \infty} \sum_{i=1}^{t-1}\left|\alpha_{\ell}(i, t, q) d_{\ell}(i)\right| \geq \frac{\left|x_{\ell}^{*}\right|}{2 \lambda_{q}}
$$

Substituting the asymptotic lower bound on the inner sum in (B.9) from (B.14), and using (B.7), we obtain

$$
\liminf _{t \rightarrow \infty} \mathbb{E}\left[\|\mathbf{e}(t)\|^{2}\right] E_{\text {com }}(t) \geq \sum_{m, q, \ell=1}^{n} \frac{a_{m, \ell}^{2} p_{m, q}^{2} \sigma_{\ell, m}^{2}\left|x_{\ell}^{*}\right|^{2}}{2 \lambda_{q}^{2}}
$$

Theorem 1 is proven. 


\section{Appendix C}

\section{Proof of Lemma B.1}

First, we derive the lower bound of $\sum_{i=t_{\ell}}^{t-1}|w(i)|$, where

$$
w(i)=\left(x_{\ell}^{*}+e_{i n, \ell}(i-1)\right)\left(1-\gamma_{\ell}(i)\right) \alpha_{\ell}(i, t, q) .
$$

We first show that an iteration number $t_{\ell}$ exists such that $\left|x_{\ell}^{*}+e_{i n, \ell}(i-1)\right| \geq(1-$ $\left.\delta_{6}\right)\left|x_{\ell}^{*}\right|$, for $i \geq t_{\ell}$. It is shown in Appendix A that norm $\left\|\mathbf{e}_{i n}(i)\right\|$ converges to zero as $i \rightarrow \infty$. Therefore, there exists an iteration number $t_{\ell}$ such that

$$
\left\|\mathbf{e}_{i n}(i-1)\right\| \leq \delta_{6}\left|x_{\ell}^{*}\right|
$$

for any $i \geq t_{\ell}$ and $x_{\ell}^{*} \neq 0$, and thus,

$$
\left|x_{\ell}^{*}+e_{i n, \ell}(i-1)\right| \geq\left|x_{\ell}^{*}\right|-\left\|\mathbf{e}_{i n}(i-1)\right\| \geq\left(1-\delta_{6}\right)\left|x_{\ell}^{*}\right|,
$$


where the first step is due to the triangle inequality and the fact that $\left|e_{i n, \ell}(i-1)\right| \leq$ $\left\|\mathbf{e}_{i n}(i-1)\right\|$. For $i \geq t_{\ell}$, we have

$$
\begin{aligned}
\sum_{i=t_{\ell}}^{t-1}|w(i)| & \geq\left(1-\delta_{6}\right)\left|x_{\ell}^{*}\right| \sum_{i=t_{\ell}}^{t-1}\left|\left(1-\gamma_{\ell}(i)\right) \alpha_{\ell}(i, t, q)\right| \\
& \geq\left(1-\delta_{6}\right)\left|x_{\ell}^{*}\right|\left|\sum_{i=t_{\ell}}^{t-1}\left(1-\gamma_{\ell}(i)\right) \alpha_{\ell}(i, t, q)\right|,
\end{aligned}
$$

where the last inequality is due to the triangle inequality.

Here, we compute the inner sum in (C.1). Recall that

$$
\alpha_{\ell}(i, t, q)=\sum_{j=i+1}^{t} b_{q}(j, t) \prod_{k=i+1}^{j-1} \gamma_{\ell}(k) .
$$

We have

$$
\begin{aligned}
\sum_{i=t_{\ell}}^{t-1}\left(1-\gamma_{\ell}(i)\right) \alpha_{\ell}(i, t, q) & =\sum_{i=t_{\ell}}^{t-1}\left(1-\gamma_{\ell}(i)\right) \sum_{j=i+1}^{t-1} b_{q}(j, t) \prod_{k=i+1}^{j-1} \gamma_{\ell}(k) \\
& =\sum_{j=t_{\ell}+1}^{t} b_{q}(j, t) \sum_{i=t_{\ell}}^{t-1} \prod_{k=i+1}^{j-1} \gamma_{\ell}(k)\left(1-\gamma_{\ell}(i)\right) \\
& =\sum_{j=t_{\ell}+1}^{t} b_{q}(j, t)\left(1-\prod_{k=t_{\ell}}^{j-1} \gamma_{\ell}(k)\right) \\
& =\sum_{j=t_{\ell}+1}^{t} b_{q}(j, t)-\gamma_{\ell}\left(t_{\ell}\right) \alpha_{\ell}\left(t_{\ell}, t, q\right),
\end{aligned}
$$

where the second step is the result of changing the order of summation. Substituting (C.3) to (C.1), we obtain

$$
\sum_{i=t_{\ell}}^{t-1}\left|\frac{w(i)}{\left(1-\delta_{6}\right) x_{\ell}^{*}}\right| \geq\left|\sum_{j=t_{\ell}+1}^{t} b_{q}(j, t)-\gamma_{\ell}\left(t_{\ell}\right) \alpha_{\ell}\left(t_{\ell}, t, q\right)\right|
$$




$$
\geq\left|\sum_{j=t_{\ell}+1}^{t} b_{q}(j, t)\right|-\left|\alpha_{\ell}\left(t_{\ell}, t, q\right)\right|,
$$

where the last inequality is due to the triangle inequality, and the fact that $0 \leq \gamma_{\ell}\left(t_{\ell}\right) \leq$ 1. Here, we compute the lower bound on the right-hand side of (C.4). We need the following lemma which has been proven in Appendix F.

Lemma C.1 For any positive $\lambda_{q}$, there holds

$$
\sum_{i=1}^{t} b_{q}(j, t)=\lambda_{q}^{-1}\left(1-\prod_{j=1}^{t}\left(1-\frac{\lambda_{q}}{j^{\beta}}\right)\right) .
$$

Consider the first term on the right-hand side of (C.4). It follows from Lemma C.1 that

$$
\begin{aligned}
\sum_{j=t_{\ell}+1}^{t} b_{q}(j, t) & =\sum_{j=1}^{t} b_{q}(j, t)-\sum_{j=1}^{t_{\ell}} b_{q}(j, t) \\
& =\sum_{j=1}^{t} b_{q}(j, t)-\prod_{j=t_{\ell}+1}^{t}\left(1-\frac{\lambda_{q}}{j^{\beta}}\right) \sum_{j=1}^{t_{\ell}} b_{q}\left(j, t_{\ell}\right) \\
& =\lambda_{q}^{-1}\left(1-\prod_{j=t_{\ell}+1}^{t}\left(1-\frac{\lambda_{q}}{j^{\beta}}\right)\right)
\end{aligned}
$$

Now consider the second term on the right-hand side of (C.4). We have

$$
\begin{aligned}
\left|\alpha_{\ell}\left(t_{\ell}, t, q\right)\right| & \leq \sqrt{\psi_{\ell}^{2}\left(t_{\ell}\right) \sum_{i=1}^{t-1}\left(\frac{\alpha_{\ell}(i, t, q)}{\psi_{\ell}(i)}\right)^{2}} \\
& \leq \psi_{\ell}\left(t_{\ell}\right) \sqrt{\sum_{i=1}^{t-1}\left(\frac{\alpha_{\ell}(i, t, q)}{\psi_{\ell}(i)}\right)^{2}} .
\end{aligned}
$$


Substituting (C.5) and (C.6) in (C.4), and using the Lemma 1 and Lemma G.1 (Appendix G), we obtain (B.12).

It remains to derive the upper bound of $\sum_{i=t_{\ell}}^{t-1}|z(i)|$, where

$$
z(i)=\frac{\alpha_{\ell}(i, t, q)}{i^{\beta}}\left\langle[\mathbf{A}]_{\ell}, \mathbf{e}_{i n}(i-1)\right\rangle
$$

From the Cauchy-Schwartz inequality, we have

$$
\sum_{i=t_{\ell}}^{t-1}|z(i)| \leq\|\mathbf{A}\| \max _{i \geq t_{\ell}} \alpha_{\ell}(i, t, q) \sum_{i=t_{\ell}}^{t-1} \frac{\left\|\mathbf{e}_{i n}(i-1)\right\|}{i^{\beta}} .
$$

We first derive the upper bound on $\alpha_{\ell}(i, t, q)$. Notice that $0 \leq \gamma_{\ell}(k) \leq 1$. Therefore, for $i \geq\left\lfloor\sqrt[\beta]{\lambda_{q}}\right\rfloor$, we have

$$
\begin{aligned}
\alpha_{\ell}(i, t, q) & \leq \sum_{j=i+1}^{t} \frac{1}{j^{\beta}} \prod_{k=j+1}^{t}\left(1-\frac{\lambda_{q}}{k^{\beta}}\right) \\
& \leq e^{\frac{-\lambda_{q}(t+1)^{1-\beta}}{1-\beta}} \sum_{j=i+1}^{t} \frac{e^{\frac{\lambda_{q}(j+1)^{1-\beta}}{1-\beta}}}{j^{\beta}}
\end{aligned}
$$

where the last step is due to Lemma G.1 in Appendix G. The summand in (C.8) is an increasing function of iteration number $i$. Therefore, the inner sum of (C.8) is upper bounded by

$$
\begin{aligned}
\sum_{j=i+1}^{t} \frac{e^{\frac{\lambda_{q}(j+1)^{1-\beta}}{1-\beta}}}{j^{\beta}} & \leq \frac{e^{\frac{\lambda_{q}(t+1)^{1-\beta}}{1-\beta}}}{t^{\beta}}+\frac{1}{1-\beta} \int_{(i+2)^{1-\beta}}^{(t+1)^{1-\beta}} \frac{e^{\frac{\lambda_{q} v}{1-\beta}}}{\left(1-v^{\frac{-1}{1-\beta}}\right)^{\beta}} d v \\
& \leq \frac{e^{\frac{\lambda_{q}(t+1)^{1-\beta}}{1-\beta}}}{t^{\beta}}+\frac{\left(1-(i+2)^{-1 / 1-\beta}\right)^{-\beta}}{1-\beta} \int_{(i+2)^{1-\beta}}^{(t+1)^{1-\beta}} e^{\frac{\lambda_{q} v}{1-\beta}} d v
\end{aligned}
$$




$$
\leq\left(t_{\ell}^{-\beta}+\left(1-t_{\ell}^{-1 / 1-\beta}\right)^{-\beta} \lambda_{q}^{-1}\right) e^{\frac{\lambda_{q}(t+1)^{1-\beta}}{1-\beta}}
$$

where the third step is due to the Holder's inequality. Substituting (C.9) in (C.8), we obtain

$$
\alpha_{\ell}(i, t, q) \leq c_{4}\left(t_{\ell}\right)
$$

where $c_{4}\left(t_{\ell}\right) \triangleq t_{\ell}^{-\beta}+\left(1-t_{\ell}^{-1 / 1-\beta}\right)^{-\beta} \lambda_{q}^{-1}$. Substituting (C.10) in (C.7), and using the asymptotic upper bound on $\left\|\mathbf{e}_{i n}(i)\right\|$, equation (2.11), we prove that there exists an iteration number $t_{\ell}$ such that

$$
\begin{aligned}
\sum_{i=t_{\ell}}^{t-1}|z(i)| & \leq \lambda_{\max } c_{4}\left(t_{\ell}\right) \sum_{i=t_{\ell}}^{\infty} \frac{e^{\frac{-\lambda_{\min } i^{1-\beta}}{1-\beta}}}{i^{\beta}} \\
& \leq \frac{\lambda_{\max } c_{4}\left(t_{\ell}\right)\left(1-t_{\ell}^{-1 / 1-\beta}\right)}{1-\beta} e^{\frac{-\lambda_{\min }\left(t_{\ell}-1\right)^{1-\beta}}{1-\beta}}
\end{aligned}
$$

Notice that the right-hand side of inequality (C.11) is a decreasing function of $t_{\ell}$. Therefore, we can choose $t_{\ell}$ large enough to satisfy $\lim _{t \rightarrow \infty} \sum_{i=t_{\ell}}^{t-1}|z(i)| \leq \delta_{7}$, for any positive $\delta_{7}$. The lemma is proven. 


\section{Appendix D}

\section{Proof of (2.16)}

We need the following lemma to derive an upper bound on $\alpha(i, t, q)$.

Lemma D.1 For positive $\kappa, \lambda_{q}, \delta_{1}, 0<\beta<1$, and $t>i>\zeta$, there holds

$$
\int_{i}^{t} \frac{e^{\frac{\lambda_{q} v}{1-\beta}}}{\left(v^{\frac{1}{1-\beta}}-2\right)^{\kappa}} d v \leq \frac{\left(1+\delta_{1}\right)(1-\beta) e^{\frac{\lambda_{q} t}{1-\beta}}}{\lambda_{q}\left(t^{\frac{1}{1-\beta}}-2\right)^{\kappa}}
$$

where $\zeta \triangleq\left[\max \left\{\left(\lambda_{q} \delta_{1} \kappa^{-1}\right)^{\frac{1-\beta}{\beta}}, 4 \lambda_{q}^{-1} \delta_{1}^{-1} \kappa, 2^{1-\beta}\right\}\right]$.

Proof: Define

$$
r(t) \triangleq \frac{\left(1+\delta_{1}\right)(1-\beta) e^{\frac{\lambda_{q} t}{1-\beta}}}{\lambda_{q}\left(t^{\frac{1}{1-\beta}}-2\right)^{\kappa}}-\int_{i}^{t} \frac{e^{\frac{\lambda_{q} v}{1-\beta}}}{\left(v^{\frac{1}{1-\beta}}-2\right)^{\kappa}} d v .
$$


Notice that $r(i) \geq 0$ by definition. Therefore, it is enough to show that $r(t)$ is an increasing function of $t$, or equivalently

$$
\frac{d r(t)}{d t}>0 \Longleftrightarrow\left[\delta_{1}-\frac{2 \kappa t^{\frac{\beta}{1-\beta}}}{\lambda_{q}\left(t^{\frac{1}{1-\beta}}-2\right)}\right] \frac{e^{\frac{\lambda_{q} t}{1-\beta}}}{\left(t^{\frac{1}{1-\beta}}-2\right)^{\kappa}}>0
$$

which holds when $t>\zeta$. The lemma is proven.

By definition of $\alpha(i, t, q)$, and Lemma G.1 in Appendix G, we have

$$
\begin{aligned}
\alpha(i, t, q) & =\sum_{j=i+1}^{t} \frac{1}{j^{\beta}} \prod_{k=j+1}^{t}\left(1-\frac{\lambda_{q}}{k^{\beta}}\right) \prod_{k=i+1}^{j-1}\left(\frac{k-1}{k}\right)^{\kappa} \\
& \leq i^{\kappa} e^{\frac{-\lambda_{q}(t+1)^{1-\beta}}{1-\beta}} \sum_{j=i+1}^{t} \eta(j)
\end{aligned}
$$

where

$$
\eta(j) \triangleq \frac{e^{\frac{\lambda_{q}(j+1)^{1-\beta}}{1-\beta}}}{(j-1)^{\kappa+\beta}} .
$$

Notice that the inner sum in (D.1) is upper bounded by

$$
\sum_{j=i+1}^{t} \eta(j) \leq \sum_{j=1}^{\zeta-1} \eta(j)+\sum_{j=\zeta}^{t} \eta(j)
$$

The first term of (D.2) is bounded, and the second term of (D.2) is upper bounded by

$$
\begin{aligned}
\sum_{j=\zeta}^{t} \eta(j) & \leq \int_{\zeta+1}^{t+1} \frac{e^{\frac{\lambda q u^{1-\beta}}{1-\beta}}}{(u-2)^{\kappa+\beta}} d u \\
& \leq \frac{1}{1-\beta} \int_{(\zeta+1)^{1-\beta}}^{(t+1)^{1-\beta}} \frac{e^{\frac{\lambda_{q} v}{1-\beta}}}{\left(v^{\frac{1}{1-\beta}}-2\right)^{\kappa}} d v
\end{aligned}
$$




$$
\leq \frac{\left(1+\delta_{1}\right) e^{\frac{\lambda_{q}(t+1)^{1-\beta}}{1-\beta}}}{\lambda_{q}(t-1)^{\kappa}}
$$

where the second step is obtained by changing variable $v=u^{1-\beta}$, and the last step is due to Lemma D.1. Substituting (D.2)-(D.3) in (D.1), and choosing $\delta_{1}=1$, we obtain (2.16). 


\section{Appendix E}

\section{Upper Bound on Communication}

\section{Energy for the Isotropic Universal}

\section{Design}

Recall that $E_{\text {com }}(t)=E_{\text {in }}(t)+E_{c h}(t)$. We derive an upper bound on communication energy by computing upper bounds on $E_{i n}(t)$ and $E_{c h}(t)$. Consider $E_{i n}(t)$ in (B.3), we have

$$
\begin{aligned}
E_{\text {in }}(t) & \leq 2 \sum_{i=1}^{t-1} \hat{\psi}^{2}(i)(1-\hat{\gamma}(i))^{2}\left\|\mathbf{x}^{*}+\mathbf{e}_{i n}(i-1)\right\|^{2} \\
& +2 \sum_{i=1}^{t-1} \frac{\hat{\psi}^{2}(i)}{i^{2 \beta}}\|\mathbf{A}\|^{2}\left\|\mathbf{e}_{i n}(i-1)\right\|^{2} .
\end{aligned}
$$

Here, we compute an upper bound on the first term of (E.1). It is shown in Appendix A that norm $\left\|\mathbf{e}_{i n}(i)\right\|$ converges to zero as $i \rightarrow \infty$. Therefore, there exists an iteration 
number $t_{d}$ such that

$$
\left\|\mathbf{e}_{i n}(i-1)\right\| \leq \frac{1}{2}\left\|\mathbf{x}_{\ell}^{*}\right\|
$$

for any $i \geq t_{d}$, and non-zero $\mathbf{x}^{*}$, and thus,

$$
\left\|\mathbf{x}^{*}+\mathbf{e}_{i n}(i-1)\right\| \leq\left\|\mathbf{x}^{*}\right\|+\left\|\mathbf{e}_{i n}(i-1)\right\| \leq\left(1+\frac{1}{2}\right)\left\|\mathbf{x}^{*}\right\|
$$

where the first step is due to the triangle inequality. Therefore,

$$
\sum_{i=1}^{t-1} \hat{\psi}^{2}(i)(1-\hat{\gamma}(i))^{2}\left\|\mathbf{x}^{*}+\mathbf{e}(i n)(i-1)\right\|^{2} \leq \mathcal{O}(1)+\frac{9}{4}\left\|\mathbf{x}^{*}\right\|^{2} \sum_{i=t_{d}}^{t-1} \hat{\psi}^{2}(i)(1-\hat{\gamma}(i))^{2}
$$

It follows from the Taylor expansion of $\left(1-i^{-1}\right)^{\kappa}$ that

$$
\begin{aligned}
\sum_{i=t_{d}}^{t-1} \hat{\psi}^{2}(i)(1-\hat{\gamma}(i))^{2} & =\sum_{i=t_{d}}^{t-1} i^{1+\kappa}\left(1-\left(1-i^{-1}\right)^{\kappa}\right)^{2} \\
& =\sum_{i=t_{d}}^{t-1}\left(\kappa^{2} i^{\kappa-1}+o\left(i^{\kappa-1}\right)\right) \\
& \leq \kappa t^{\kappa}+o\left(t^{\kappa}\right) .
\end{aligned}
$$

We then compute an upper bound on the second term of (E.1). It follows from the convergence rate of $\left\|e_{i n}(i)\right\|$ in $(2.11)$ that

$$
\sum_{i=1}^{t-1} \frac{\hat{\psi}^{2}(i)}{i^{2 \beta}}\|\mathbf{A}\|^{2}\left\|\mathbf{e}_{i n}(i-1)\right\|^{2}=\mathcal{O}(1)
$$


We conclude from (E.1)-(E.2) that

$$
E_{\text {in }}(t) \leq \frac{9}{2} \kappa\left\|\mathbf{x}^{*}\right\|^{2} t^{\kappa}+o\left(t^{\kappa}\right)
$$

Similar to (E.1), we have

$$
\begin{aligned}
E_{c h}(t) & \leq 2 \sum_{i=1}^{t-1} \hat{\psi}^{2}(i)(1-\hat{\gamma}(i))^{2} \mathbb{E}\left[\left\|\mathbf{e}_{c h}(i-1)\right\|^{2}\right] \\
& +2 \sum_{i=1}^{t-1}\left\|\frac{\hat{\psi}(i) \mathbf{A}}{i^{\beta}}\right\|^{2} \mathbb{E}\left[\left\|\mathbf{e}_{c h}(i-1)\right\|^{2}\right] \\
& +2 \sum_{i=1}^{t-1} \mathbb{E}\left[\left\|\frac{\hat{\psi}(i) \mathbf{v}(i-1)}{i^{\beta}}\right\|^{2}\right],
\end{aligned}
$$

where $\mathbb{E}\left[\left\|\mathbf{e}_{c h}(i)\right\|^{2}\right]$ is the contribution of accumulated channel noise on mean squared error at $i$-th iteration. We compute an upper bound on the right-hand side of (E.4) term by term. Substituting $\mathbb{E}\left[\left\|\mathbf{e}_{c h}(i)\right\|^{2}\right]$ from (2.18), and the Taylor expansion of $\left(1-i^{-1}\right)^{\kappa}$, we obtain

$$
\begin{aligned}
\sum_{i=1}^{t-1} \hat{\psi}^{2}(i)(1-\hat{\gamma}(i))^{2} \mathbb{E}\left[\left\|\mathbf{e}_{c h}(i-1)\right\|^{2}\right] & =\mathcal{O}(\log (t)) \\
\sum_{i=1}^{t-1} \hat{\psi}^{2}(i)\left\|\frac{\mathbf{A}}{i^{\beta}}\right\|^{2} \mathbb{E}\left[\left\|\mathbf{e}_{c h}(i-1)\right\|^{2}\right] & =\mathcal{O}\left(t^{2(1-\beta)}\right) .
\end{aligned}
$$

Now consider the third term of (E.4). Notice that the channel noise is temporally and spatially uncorrelated, therefore,

$$
\mathbb{E}\left[\|\mathbf{v}(i-1)\|^{2}\right]=\sum_{m, \ell=1}^{n} a_{m, \ell}^{2} \sigma_{\ell, m}^{2} \sum_{j=1}^{i-1} \frac{j^{\kappa-1}}{i^{2 \kappa}}
$$




$$
=\left(\sum_{m, \ell=1}^{n} \frac{a_{m, \ell}^{2} \sigma_{\ell, m}^{2}}{\kappa}\right) i^{-\kappa} .
$$

Therefore, the last term of (E.4) is $\mathcal{O}\left(t^{2(1-\beta)}\right)$, and hence

$$
E_{c h}(t)=\mathcal{O}\left(t^{2(1-\beta)}\right)
$$

Equation (2.19) follows from (E.3) and (E.5). 


\section{Appendix F}

\section{Proof of Lemma C.1}

Proof: Define

$$
k(t) \triangleq \sum_{i=1}^{t} b_{q}(i, t)=\sum_{i=1}^{t} \frac{1}{i^{\beta}} \prod_{j=i+1}^{t}\left(1-\frac{\lambda_{q}}{j^{\beta}}\right)
$$

Notice that

$$
k(t)=\left(1-\frac{\lambda_{q}}{t^{\beta}}\right) k(t-1)+\frac{1}{t^{\beta}} .
$$

Define $h(t) \triangleq \lambda_{q}^{-1}-k(t)$, and substitute the value of $k(t)$ in terms of $k(t-1)$ to obtain

$$
\begin{aligned}
h(t) & \triangleq \lambda_{q}^{-1}-\left(1-\frac{\lambda_{q}}{t^{\beta}}\right) k(t-1)-\frac{1}{t^{\beta}} \\
& =\lambda_{q}^{-1}-\left[\left(1-\frac{\lambda_{q}}{t^{\beta}}\right)\left(\lambda_{q}^{-1}-h(t-1)\right)+\frac{1}{t^{\beta}}\right] \\
& =\left(1-\frac{\lambda_{q}}{t^{\beta}}\right) h(t-1)
\end{aligned}
$$




$$
=\prod_{j=2}^{t}\left(1-\frac{\lambda_{q}}{j^{\beta}}\right) h(1),
$$

where

$$
h(1)=\lambda_{q}^{-1}-k(1)=\lambda_{q}^{-1}-1=-\frac{1-\lambda_{q}}{\lambda_{q}} .
$$

The lemma is proven. 


\section{Appendix G}

\section{Proof of Lemma G.1}

Lemma G.1 For any $i, t>\lfloor\sqrt[\beta]{\lambda}\rfloor, 0<\beta<1$, and $\lambda>0$, there holds

$$
\prod_{j=i}^{t-1}\left(1-\frac{\lambda}{j^{\beta}}\right) \leq e^{\frac{-\lambda\left(t^{1-\beta}-i^{1-\beta}\right)}{1-\beta}} .
$$

Proof: Note that for $i>\lfloor\sqrt[\beta]{\lambda}\rfloor$, we have

$$
\left|\prod_{j=i}^{t-1}\left(1-\frac{\lambda}{j^{\beta}}\right)\right|=e^{\sum_{j=i}^{t-1} \log \left(1-\frac{\lambda}{j^{\beta}}\right)} .
$$

Use the fact that $\log (1+x) \leq x, \forall x>-1$, to compute an upper bound on the inner sum in (G.1):

$$
\sum_{j=i}^{t-1} \log \left(1-\frac{\lambda}{j^{\beta}}\right) \leq \sum_{j=i}^{t-1} \frac{-\lambda}{j^{\beta}} \leq \frac{\lambda}{1-\beta}\left[i^{1-\beta}-t^{1-\beta}\right] .
$$


Substitute (G.2) in (G.1) to prove the lemma.

Consider a symmetric positive definite matrix $\mathbf{A}$, with eigenvalues $\lambda_{q}, q \in\{1, \ldots, n\}$, and define

$$
c_{3}(\lambda) \triangleq \prod_{j=1}^{\lfloor\sqrt[\beta]{\lambda}\rfloor} \frac{\lambda-j^{\beta}}{j^{\beta}} e^{\frac{\lambda(\lfloor\beta \lambda\rfloor+1)^{1-\beta}}{1-\beta}} .
$$

Corollary G.1 For $\mathbf{D}(i)=\prod_{j=1}^{i-1}\left(\mathbf{I}-\frac{\mathbf{A}}{j^{\beta}}\right)$, there holds

$$
\|\mathbf{D}(i)\| \leq c_{3}\left(\lambda_{\min }\right) e^{\frac{-\lambda_{\min } i^{1-\beta}}{1-\beta}}
$$

when $i$ satisfies

$$
i>\max _{\lambda_{q} \neq \lambda_{\min }}\left\{\frac{(1-\beta) \log \left(\frac{c_{3}\left(\lambda_{q}\right)}{c_{3}\left(\lambda_{\min }\right)}\right)}{\lambda_{q}-\lambda_{\min }}, \sqrt[\beta]{\lambda_{\max }}\right\} .
$$

Proof: Since matrix A is a symmetric matrix, there holds (from Lemma G.1)

$$
\begin{aligned}
\|\mathbf{D}(i)\| & =\max _{q \in\{1, \ldots, n\}}\left|\prod_{j=1}^{i-1}\left(1-\frac{\lambda_{q}}{j^{\beta}}\right)\right| \\
& \leq \max _{q \in\{1, \ldots, n\}} c_{3}\left(\lambda_{q}\right) e^{\frac{-\lambda_{q} i^{1-\lambda_{q}}}{1-\beta}} \\
& =c_{3}\left(\lambda_{\min }\right) e^{\frac{-\lambda_{\min } i^{1-\beta}}{1-\beta}}
\end{aligned}
$$

where the last step holds when condition (G.3) satisfies. The lemma is proven. 


\section{Appendix H}

\section{Proof of Lemma 1}

Similar to the proof of proposition 5.1 in [54], we prove that under assumptions provided in Lemma 1, then it holds

$$
\begin{aligned}
\mathbb{E}\left[f(\mathbf{x}(t))-f\left(\mathbf{x}^{*}\right)\right] & \leq A n \alpha^{2 t} \\
\mathbb{E}\left[\left\|\mathbf{x}(t)-\mathbf{x}^{*}\right\|^{2}\right] & \leq \frac{2 A n}{L} \alpha^{2 t}
\end{aligned}
$$

We use induction on $t$. We first show that inequality (H.1) holds for $t=0$. Since $f(\mathbf{x})$ is convex, we have

$$
\begin{aligned}
f(\mathbf{x}(0))-f\left(\mathbf{x}^{*}\right) & \leq\left\langle\nabla f(\mathbf{x}(0)), \mathbf{x}(0)-\mathbf{x}^{*}\right\rangle \\
& \leq\|\nabla f(\mathbf{x}(0))\|\left\|\mathbf{x}(0)-\mathbf{x}^{*}\right\| \\
& \leq M L\left\|\mathbf{x}(0)-\mathbf{x}^{*}\right\|^{2}=M L n
\end{aligned}
$$


where the last two step is due to the fact that $f(\mathbf{x}) \in \mathcal{F}_{M, L}$, and $\mathbf{x}(0)-\mathbf{x}^{*} \in[-1,1]^{n}$. Therefore, inequality (H.1) holds for $t=0$ as long as $A$ is chosen larger than $M L$.

Suppose inequality (H.1) holds for non-negative $t$. For strongly convex function $f(\mathbf{x})$, as it is shown in [71], we have

$$
\begin{aligned}
f(\mathbf{x}(t)) & \geq f\left(\mathbf{x}^{*}\right)+\left\langle\nabla f\left(\mathbf{x}^{*}, \mathbf{x}(t)-\mathbf{x}^{*}\right\rangle+\frac{L}{2}\left\|\mathbf{x}(t)-\mathbf{x}^{*}\right\|^{2}\right. \\
& \geq f\left(\mathbf{x}^{*}\right)+\frac{L}{2}\left\|\mathbf{x}(t)-\mathbf{x}^{*}\right\|^{2},
\end{aligned}
$$

where the last inequality is due to necessary and sufficient condition for optimality of $\mathrm{x}^{*}$. Therefore, inequality (H.2) is valid as

$$
\mathbb{E}\left[\left\|\mathbf{x}(t)-\mathbf{x}^{*}\right\|^{2}\right] \leq \frac{2}{L} \mathbb{E}\left[f(\mathbf{x}(t))-f\left(\mathbf{x}^{*}\right)\right] \leq \frac{2 A n}{L} \alpha^{2 t} .
$$

Here, we prove (H.1) for $t+1$ to complete the induction. Define

$$
J(\mathbf{x}(t+1)) \triangleq\langle\mathbf{g}(\mathbf{x}(t)), \mathbf{x}(t+1)-\mathbf{x}(t)\rangle+\frac{L M}{2}\|\mathbf{x}(t+1)-\mathbf{x}(t)\|^{2} .
$$

It is shown for strongly convex function $f(\mathbf{x})$ in [71] that

$$
\begin{aligned}
f(\mathbf{x}(t+1))-f(\mathbf{x}(t)) & \leq\left\langle\nabla f(\mathbf{x}(t), \mathbf{x}(t+1)-\mathbf{x}(t)\rangle+\frac{L M}{2}\|\mathbf{x}(t+1)-\mathbf{x}(t)\|^{2}\right. \\
& \leq\langle\mathbf{g}(\mathbf{x}(t)), \mathbf{x}(t+1)-\mathbf{x}(t)\rangle+\frac{L M}{2}\|\mathbf{x}(t+1)-\mathbf{x}(t)\|^{2} \\
& +\| \mathbf{g}(\mathbf{x}(t))-\nabla f(\mathbf{x}(t)\|\| \mathbf{x}(t+1)-\mathbf{x}(t) \| \\
& =J(\mathbf{x}(t+1))+\| \mathbf{g}(\mathbf{x}(t))-\nabla f(\mathbf{x}(t)\|\| \mathbf{x}(t+1)-\mathbf{x}(t) \|
\end{aligned}
$$


where the second inequity is due to the Schwartz inequality, and the last equality follows from the definition of $J(\mathbf{y})$. Notice that $\mathbf{x}(t+1)$ minimizes $\left\|\mathbf{y}-\mathbf{x}(t)+\frac{1}{L M} \mathbf{g}(\mathbf{x}(t))\right\|^{2}$ over $\mathbf{y} \in[0,1]^{n}$ by the definition of the projection, or equivalently,

$$
\mathbf{x}(t+1)=\arg \min J(\mathbf{y}),
$$

and thus, we have

$$
\begin{aligned}
J(\mathbf{x}(t+1)) & \leq J\left(\mathbf{x}(t)+\frac{1}{M}\left(\mathbf{x}^{*}-\mathbf{x}(t)\right)\right) \\
& \leq \frac{1}{M}\left\langle\mathbf{g}(\mathbf{x}(t)), \mathbf{x}^{*}-\mathbf{x}(t)\right\rangle+\frac{L}{2 M}\left\|\mathbf{x}^{*}-\mathbf{x}(t)\right\|^{2} \\
& \leq \frac{1}{M}\left(\left\langle\nabla f\left(\mathbf{x}(t), \mathbf{x}^{*}-\mathbf{x}(t)\right\rangle+\frac{L}{2}\left\|\mathbf{x}^{*}-\mathbf{x}(t)\right\|^{2}\right)\right. \\
& +\frac{1}{M} \| \mathbf{g}(\mathbf{x}(t))-\nabla f\left(\mathbf{x}(t)\|\| \mathbf{x}^{*}-\mathbf{x}(t) \|\right. \\
& \leq \frac{1}{M}\left(f(\mathbf{x}(t))-f\left(\mathbf{x}^{*}\right)\right) \\
& +\frac{1}{M} \| \mathbf{g}(\mathbf{x}(t))-\nabla f\left(\mathbf{x}(t)\|\| \mathbf{x}^{*}-\mathbf{x}(t) \|,\right.
\end{aligned}
$$

where the last inequality follows from (H.4). Define $\delta f(t) \triangleq f(\mathbf{x}(t))-f\left(\mathbf{x}^{*}\right)$, and substitute (H.5) into (H.4) to obtain

$$
\begin{aligned}
\delta f(t) & \leq\left(1-\frac{1}{M}\right) \delta f(t+1) \\
& +\| \mathbf{g}(\mathbf{x}(t))-\nabla f(\mathbf{x}(t)\|\| \mathbf{x}(t+1)-\mathbf{x}(t) \| \\
& +\frac{1}{M} \| \mathbf{g}(\mathbf{x}(t))-\nabla f\left(\mathbf{x}(t)\|\| \mathbf{x}(t)-\mathbf{x}^{*} \|\right.
\end{aligned}
$$

It follows from update formula for gradient projection algorithm (3.4) and triangle in- 
equality that

$$
\begin{aligned}
\|\mathbf{x}(t+1)-\mathbf{x}(t)\| & \leq\left\|\frac{\mathbf{g}(\mathbf{x}(t))}{L M}\right\| \\
& \leq \frac{\| \mathbf{g}(\mathbf{x}(t))-\nabla f(\mathbf{x}(t)\|+\| \nabla f(\mathbf{x}(t) \|}{L M} \\
& \leq \frac{\| \mathbf{g}(\mathbf{x}(t))-\nabla f(\mathbf{x}(t) \|}{L M}+\left\|\mathbf{x}(t)-\mathbf{x}^{*}\right\|,
\end{aligned}
$$

where the last inequity holds for $f(\mathbf{x}) \in \mathcal{F}_{M, L}$. Substitute (H.7) into (H.6) to derive

$$
\begin{aligned}
\delta f(t+1) & \leq\left(1-\frac{1}{M}\right) \delta f(t)+\frac{\| \mathbf{g}(\mathbf{x}(t))-\nabla f\left(\mathbf{x}(t) \|^{2}\right.}{M L} \\
& +\left(1+\frac{1}{M}\right) \| \mathbf{g}(\mathbf{x}(t))-\nabla f\left(\mathbf{x}(t)\|\| \mathbf{x}(t)-\mathbf{x}^{*} \|\right.
\end{aligned}
$$

Therefore, from the induction hypothesis and the fact that $\mathbb{E}[x y] \leq \sqrt{\mathbb{E}[x] \mathbb{E}[y]}$, we have

$$
\mathbb{E}[\delta f(t+1)] \leq\left(1-\frac{1}{M}\right) A n \alpha^{2 t}+\frac{n \alpha^{2 t}}{L M}+\left(1+\frac{1}{M}\right) \sqrt{\frac{2 A n^{2}}{L}} \alpha^{2 t}
$$

The induction will be complete if the right-hand side of (H.8) be smaller than $A n \alpha^{2(t+1)}$, or equivalently if the following inequality holds:

$$
\left(1-\frac{1}{M}\right)+\frac{1}{L M A}+\left(1+\frac{1}{M}\right) \sqrt{\frac{2}{A L}} \leq \alpha^{2} .
$$

